\title{
Starbursts with suppressed velocity dispersion revealed in a forming cluster at $z=2.51$
}

\section{Mengyuan Xiao}

Nanjing University https://orcid.org/0000-0003-1207-5344

Tao Wang ( $\sim$ taowang@nju.edu.cn )

Nanjing University https://orcid.org/0000-0002-2504-2421

\section{David Elbaz}

CEA Saclay

Daisuke lono

National Astronomical Observatory of Japan

Xing Lu

NAOJ

Longji Bing

LAM

\section{Emanuele Daddi}

CEA Saclay https://orcid.org/0000-0002-3331-9590

\section{Benjamin Magnelli}

CEA Saclay https://orcid.org/0000-0002-6777-6490

\section{Carlos Gómez-Guijarro}

CEA Saclay https://orcid.org/0000-0002-4085-9165

\section{Frederic Bournaud}

CEA Saclay

Qiusheng Gu

Nanjing University

Shuowen Jin

Cosmic DAWN center https://orcid.org/0000-0002-8412-7951

\section{Francesco Valentino}

DARK, Copenhagen

\section{Anita Zanella}

Istituto Nazionale di Astrofisica

\section{Raphael Gobat}

Pontificia Universidad Católica de Valparaíso

\section{Sergio Martin}

European Southern Observatory https://orcid.org/0000-0001-9281-2919

\section{Gabriel Brammer}


University of Copenhagen https://orcid.org/0000-0003-2680-005X

\section{Kotaro Kohno}

Institute of Astronomy, School of Science, The University of Tokyo https://orcid.org/0000-0002-40522394

\section{Corentin Schreiber}

Astrophysics, Department of Physics, Keble Road, Oxford OX1 3RH, UK

\section{Laure Ciesla}

Laboratoire AIM-Paris-Saclay

\section{Xiaoling Yu}

NanJing University

\section{Koryo Okumura}

CEA Saclay

\section{Article}

Keywords: starbursts, galaxy clusters, suppressed velocity dispersion

Posted Date: October 15th, 2021

DOl: https://doi.org/10.21203/rs.3.rs-954128/v1

License: (c) (1) This work is licensed under a Creative Commons Attribution 4.0 International License. Read Full License 


\section{Starbursts with suppressed velocity dispersion revealed in a forming cluster at}

$z=2.51$

M.-Y. Xiao ${ }^{1,2,3}$, T. Wang ${ }^{1,3 凶}$, D. Elbaz ${ }^{2}$, D. Iono ${ }^{4,5}$, X. Lu ${ }^{4}$, L.-J. Bing ${ }^{6}$, E. Daddi ${ }^{2}$, B. Magnelli ${ }^{2}$, C. Gómez-Guijarro ${ }^{2}$, F. Bournaud $^{2}$, Q.-S. Gu ${ }^{1,3}$, S.-W. Jin ${ }^{7,8}$, F. Valentino ${ }^{7,9}$, A. Zanella ${ }^{10}$, R. Gobat ${ }^{11}$, S. Martin ${ }^{12,13}$, G. Brammer ${ }^{7,9}$, K. Kohno ${ }^{14}$, C. Schreiber ${ }^{15}$, L. Ciesla ${ }^{6}$, X.-L. Yu ${ }^{1,3}$ \& K. Okumura ${ }^{2}$

${ }^{1}$ School of Astronomy and Space Science, Nanjing University, Nanjing 210093,P. R. China

${ }^{2}$ AIM, CEA, CNRS, Université Paris-Saclay, Université Paris Diderot, Sorbonne Paris Cité, F-91191 Gif-sur-Yvette, France

${ }^{3}$ Key Laboratory of Modern Astronomy and Astrophysics (Nanjing University), Ministry of Education, Nanjing 210093, China

${ }^{4}$ National Astronomical Observatory of Japan, 2-21-1 Osawa, Mitaka, Tokyo 181-8588, Japan

${ }^{5}$ Department of Astronomical Science, SOKENDAI (The Graduate University for Advanced Studies), Mitaka, Tokyo 181-8588, Japan

${ }^{6}$ Aix Marseille Université, CNRS, LAM, Laboratoire d'Astrophysique de Marseille, Marseille, France

${ }^{7}$ Cosmic Dawn Center (DAWN), Copenhagen, Denmark

${ }^{8}$ DTU-Space, Technical University of Denmark, Elektrovej 327, DK2800 Kgs. Lyngby, Denmark

${ }^{9}$ Niels Bohr Institute, University of Copenhagen, Blegdamsvej 17, DK2100 Copenhagen Ø, Denmark

${ }^{10}$ Istituto Nazionale di Astrofisica, Vicolo dell'Osservatorio 5, I35122 Padova, Italy

${ }^{11}$ Instituto de Física, Pontificia Universidad Católica de Valparaíso, Casilla 4059, Valparaíso, Chile

${ }^{12}$ European Southern Observatory, Alonso de Córdova, 3107, Vitacura, Santiago 763-0355, Chile

${ }^{13}$ Joint ALMA Observatory, Alonso de Córdova, 3107, Vitacura, Santiago 763-0355, Chile

${ }^{14}$ Institute of Astronomy, Graduate School of Science, The University of Tokyo, 2-21-1 Osawa, Mitaka, Tokyo 181-0015, Japan

${ }^{15}$ Astrophysics, Department of Physics, Keble Road, Oxford OX1 $3 R H, U K$

$\bigotimes_{e-m a i l: t a o w a n g @ n j u . e d u . c n}$

One of the most prominent features of galaxy clusters is the presence of a dominant population of massive ellipticals in their cores ${ }^{1}$. Stellar archaeology suggests that these gigantic beasts assembled most of their stars in the early Universe via intense starbursts ${ }^{2-6}$. However, the role of dense environments and their detailed physical mechanisms in triggering starburst activities remain unknown. Here we report spatially-resolved Atacama Large Millimeter/submillimeter Array (ALMA) observations of the molecular gas, with a resolution of about 2.5 kiloparsecs, toward a forming galaxy cluster core with intense starburst galaxies at $z=2.51$. In contrast to starburst galaxies in the field often associated with galaxy mergers or highly turbulent gaseous disks, our observations show that the two starbursts in the cluster exhibit dynamically cold (rotation-dominated) gas-rich disks. Their gas disks have extremely low velocity dispersion $(\sigma \sim 20-30 \mathbf{k m}$ $\mathbf{s}^{-1}$ ), three times lower than their field counterparts at similar redshifts. The high gas fraction and suppressed velocity dispersion yield gravitationally unstable gas disks, which enables highly efficient star formation. The suppressed velocity dispersion, likely induced by accretion of co-rotating and co-planar cold gas, might serve as an essential avenue to trigger starbursts in forming galaxy clusters at high redshift.

Galaxy clusters represent the densest environments and trace the most massive dark matter halos in the Universe ${ }^{7}$. Contrary to mature clusters in the local Universe, which are dominated by massive quiescent galaxies in their cores, (proto-)clusters hosting a significant population of galaxies with intense star formation have been found at $z \gtrsim 2^{3-6}$, the peak epoch of star formation for today's massive galaxies. One of the most distant young clusters, CLJ1001 at $z_{\text {spec }}=2.51^{8}$, exhibits extended X-ray emission and encompasses an overdensity of massive galaxies similar to that in low-redshift mature clusters. However, CLJ1001 is dominated by massive star-forming galaxies, with a total star formation rate (SFR) of $\sim 3400 M_{\odot} \mathrm{yr}^{-1}$ in its 80 kiloparsecs (kpc) core (the cluster virial radius is $R_{200 \mathrm{c}} \sim 340 \mathrm{kpc}$ ). Revealing the physical origin of cluster starbursts is key to uncover the formation and quenching mechanisms of massive galaxies in clusters, a longstanding problem in extragalactic studies. With this aim, we have conducted high-resolution CO $J=3-2$ emission line $(\mathrm{CO}(3-2)$, hereafter) observations with ALMA towards the cluster core of CLJ1001 (Fig. (1) to study the gas kinematics of its member galaxies.

Four member galaxies have a $\mathrm{CO}(3-2)$ line detected with high significances $(35 \sigma, 27 \sigma, 23 \sigma$, and $10 \sigma$ for the SB1, SB2, MS1, and MS2, respectively; see Extended Data Table 11, allowing a detailed study of their kinematics. They are all massive star-forming galaxies with stellar masses of $\log \left(M_{*} / M_{\odot}\right)>10.8$ (where $M_{\odot}$ is the mass of the Sun), and are located in the central $80 \mathrm{kpc}$ region of the cluster (Fig. 11. These include two starburst galaxies (SBs) and two mainsequence galaxies (MSs), with the SBs exhibiting specific star-formation rates $\left(\mathrm{sSFR} \equiv \mathrm{SFR} / M_{*}\right)>3$ times above the star-forming main-sequence ${ }^{9}$ (SFMS, a rather tight relation between SFR and $M_{*}$ of star-forming galaxies). The intense star formation of the two SBs shows that they are rapidly building up their stellar masses at a rate of $1103_{-285}^{+385} M_{\odot} \mathrm{yr}^{-1}$ 

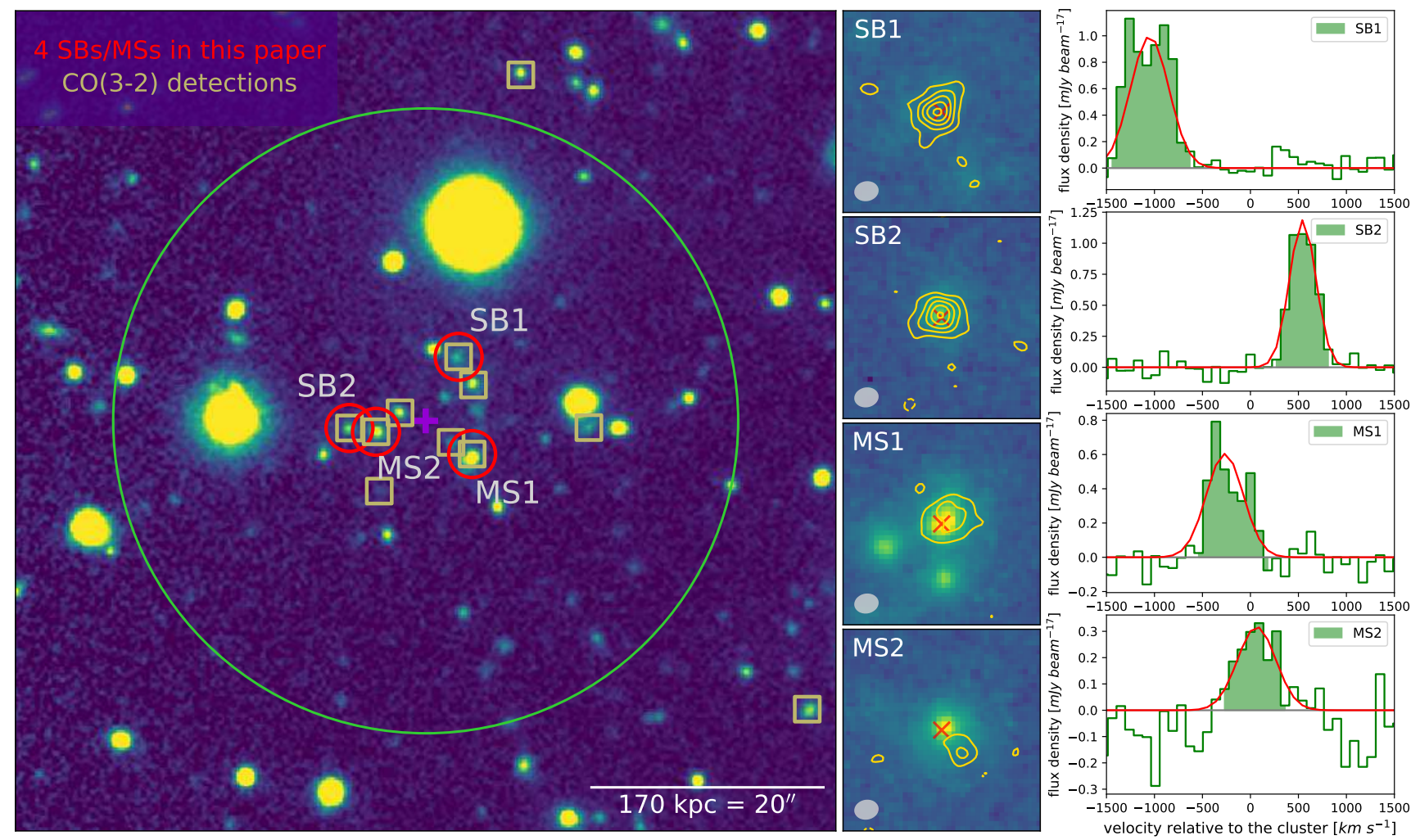

Figure 1: Galaxy cluster CLJ1001 at $z=2.51$ and the four member galaxies. Left: Sky distributions of member galaxies around the cluster center. Red circles mark the four SBs/MSs with the brightest $\mathrm{CO}(3-2)$ luminosities $(S / N \gg 10 ; S / N \sim 30$ for the two SBs) among all $\mathrm{CO}(3-2)$ detections (golden squares) in the central region of the cluster. The background is the $K_{\mathrm{S}}$-band image from UltraVista survey with a size of $70^{\prime \prime} \times 70^{\prime \prime}$. The purple cross shows the cluster center. The scale bar indicates half of the virial radius $\left(R_{200 \mathrm{c}}\right)$ of the cluster. The large green circle denotes the ALMA field of view, corresponding to the full-width at half power (FWHP) of the ALMA antennas primary beam at $98.63 \mathrm{GHz}$. Middle: Velocity-integrated intensity map (Moment 0$)$ of $\mathrm{CO}(3-2)$ (golden contours) detected by ALMA overlaid on the HST/F160W image of the four SBs/MSs. Each panel is $2.5^{\prime \prime} \times 2.5^{\prime \prime}$. The angular resolution is $0.31^{\prime \prime} \times 0.25^{\prime \prime}$ (grey filled ellipse in the bottom-left corner). The contour levels start at $\pm 3 \sigma$ and increase in steps of $\pm 3 \sigma$, where positive/negative contours are solid/dashed. The red cross in each panel denotes the centroid of the stellar emission determined from the HST/F160W image. The derived integrated fluxes are presented in Extended Data Table 1 Right: $\mathrm{CO}(3-2)$ line spectra of the four member galaxies. The CO lines are binned at $90 \mathrm{~km} \mathrm{~s}^{-1}$, and the velocity range shaded in green over which Moment 0 maps are integrated. The best-fit single Gaussian profiles are overlaid in red.

and $621_{-161}^{+217} M_{\odot} \mathrm{yr}^{-1}$ (uncertainties are the 16-84th percentile ranges of the probability distribution function; see Extended Data Table 1 and Methods for full discussion). The $\mathrm{CO}(3-2)$ emission of all four galaxies reveals continuous velocity gradients in the observed gas rotation velocity fields (Moment 1) and position-velocity (PV) diagrams (Fig. 2). The observed velocity dispersion fields (Moment 2) exhibit central dispersion peaks. These are consistent with the kinematics of rotating disks.

We adopt three commonly used methods to obtain the total molecular gas mass, $M_{\text {gas }}$, and study the gas properties of the four SBs/MSs. The molecular gas masses are independently estimated using the gas-to-dust mass ratio $\left(\delta_{\mathrm{GDR}}\right)$, the $3.2 \mathrm{~mm}$ dust continuum emission, and the $\mathrm{CO}(1-0)$ emission line ${ }^{10}$ derived with metallicity-dependent conversion factors (see Methods and Extended Data Table 2). The derived molecular gas mass fractions, $f_{\mathrm{gas}}=M_{\mathrm{gas}} /\left(M_{*}+M_{\mathrm{gas}}\right)$, for the two SBs are $~ 0.7\left(0.70_{-0.06}^{+0.09}\right.$ and $0.66_{-0.07}^{+0.10}$ based on the $\mathrm{CO}$ emission line and $0.73_{-0.08}^{+0.10}$ and $0.61_{-0.11}^{+0.13}$ based on the $3.2 \mathrm{~mm}$ dust continuum emission for the SB1 and SB2, respectively). They are twice lower for the two MSs, $f_{\text {gas }} \sim$ $0.3\left(0.29_{-0.06}^{+0.09}\right.$ and $0.25_{-0.06}^{+0.08}$ based on the $\mathrm{CO}$ emission line and $0.43_{-0.11}^{+0.13}$ and $0.18_{-0.07}^{+0.08}$ based on the $3.2 \mathrm{~mm}$ dust continuum emission for the MS1 and MS2, respectively). As a reference, the typical $f_{\text {gas }}$ of $z=2.5 \mathrm{SB}$ and MS galaxies in the field with a stellar mass of $10^{11} M_{\odot}$ is 0.7 and 0.5 , respectively ${ }^{11-13}$. It shows that the two cluster SBs have rich gas, similar to field SBs.

We investigate the kinematic properties of the molecular gas of the cluster SBs/MSs by applying a three-dimensional (3D) kinematic modelling technique ${ }^{14}$ to the $\mathrm{CO}(3-2)$ line cube. The robustness of the results given by the kinematic model has been tested by simulation (see Methods). The gas disks of the two SBs are rotation-dominated with gas rota- 


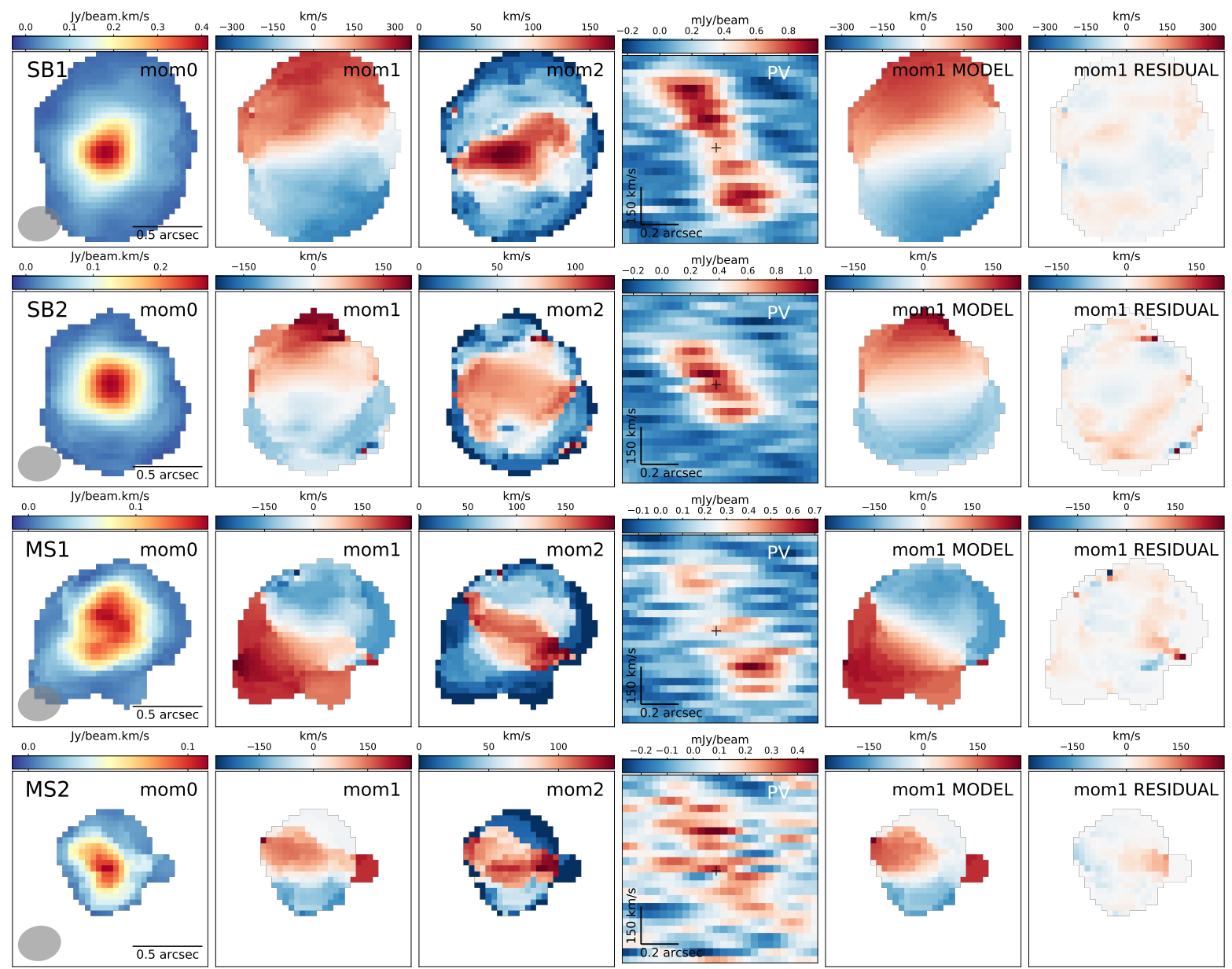

Figure 2: CO morphology and kinematics of the four cluster members at $z=2.51$. From left to right: ALMA maps $\left(1.4^{\prime \prime} \times 1.4^{\prime \prime}\right)$ of velocity-integrated $\mathrm{CO}(1-0)$ flux (Moment 0 ), velocity field (Moment 1 ), velocity dispersion (Moment 2), position-velocity (PV) diagrams along the major axis, the best-fit Moment 1 model with GalPAK ${ }^{3 D}$, and the residual between the data and the model. Note that these maps are without correction for beam-smearing. Grey filled ellipses indicate the angular resolution of $0.31^{\prime \prime} \times 0.25^{\prime \prime}$. The four member galaxies have regular rotating disks of molecular gas.

tional to random motion ratios $\left(V_{\max } / \sigma_{0}\right)$ of $16.8_{-10.4}^{+7.4}$ and $15.0_{-11.3}^{+13.0}$, while the two MSs have $V_{\max } / \sigma_{0}$ of $3.6_{-0.9}^{+1.1}$ and $2.2_{-1.2}^{+2.0}$ (Fig. 3, see Extended Data Table 3). The $V_{\max } / \sigma_{0}$ for the two SBs are more than three times higher than those for SBs and MSs in the field at the same epoch, either from observations or simulations (see Methods). Moreover, they are as high as the median ratio for disk galaxies in the local Universe. As an example, the $\mathrm{KMOS}^{3 D}$ survey $^{15}$ found a median $V_{\max } / \sigma_{0} \simeq 3$ for field MS galaxies with stellar masses of $\sim 10^{10.5} M_{\odot}$ at $\mathrm{z} \sim 2.3$. From Illustris-TNG50 simulations ${ }^{16}$, the typical star-forming galaxies, in the stellar mass range $10^{9} M_{\odot}-10^{11} M_{\odot}$, have $V_{\max } / \sigma_{0} \simeq 5 \pm 1$ at $z=2.5$ (lightblue area in Fig. 3). In addition, using $V_{\max } / \sigma_{0} \propto 1 / f_{\text {gas }}(z$, $\left.M_{*}\right)^{15}$, we get a value of $V_{\max } / \sigma_{0} \simeq 2 \pm 1$ at $z=2.5$, which decreases with increasing redshift (grey lines, light-green and pink shaded regions in Fig. 3). In general, high-redshift starforming galaxies are gas-rich and thus are believed to have a more turbulent interstellar medium than locally ${ }^{17}$. While previous studies suggest that starburst galaxies are more likely associated with mergers or interactions, which are dynami- cally hot, the two cluster SBs surprisingly host dynamically cold disks, i.e., with a large $V_{\max } / \sigma_{0}$. Recently, observations also found such phenomenon at $z>4$ with the presence of massive $\left(M_{*}>10^{10} M_{\odot}\right)$ rotation-dominated SBs (faint red points in Fig. $3{ }^{18-21}$, but no information on their environment is yet available. The rotationally supported and dynamically cold disks of the two SBs imply low gas turbulent motions, lower than that in field SBs/MSs at the same redshift (Fig. 4). Hence, the two cluster SBs appear to be weakly affected by extreme internal and/or external physical processes, such as stellar or active galactic nucleus (AGN) feedback and galaxy mergers.

To understand the triggering mechanism taking place in these two SBs, we further study the dynamical state of their disks. A rotating, symmetric gas disk is unstable to gravitational fragmentation if the Toomre parameter $Q^{22}$ is below a threshold value $Q_{\text {crit }}\left(Q_{\text {crit }}=1\right.$ for a thin gas disk and $Q_{\text {crit }}=0.67$ for a thick disk). To calculate the Toomre parameter, $Q \propto \sigma_{0} / \Sigma_{\text {gas }}$ (where $\Sigma_{\text {gas }}$ is the gas surface density), we 

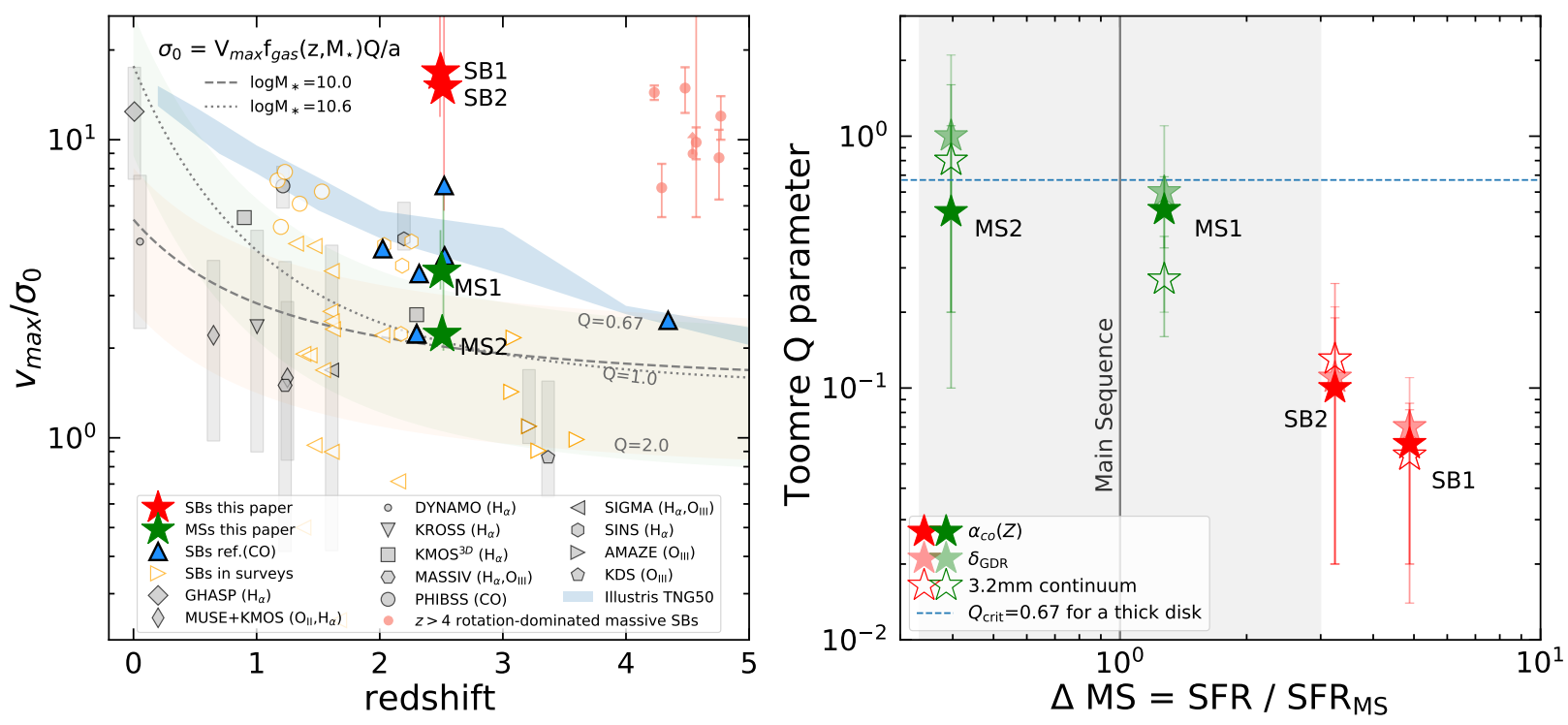

Figure 3: The two cluster SBs have dynamically cold and gravitationally unstable gas disks. Left: The ratio of the gas rotational to random motion $\left(V_{\max } / \sigma_{0}\right)$ as a function of redshift, with the comparison between the four cluster SBs/MSs and samples of observed and simulated field galaxies. The two cluster SBs and two MSs are in red and green stars, respectively, with uncertainties derived from our simulation (see Methods). Filled grey symbols with vertical bars show the median values and 16-84th percentile range of field star-forming galaxies, including molecular and ionized gas detections. When possible, we identified field starbursts (orange symbols) within these literature samples at $z>1$ as galaxies with a SFR at least 0.5 dex higher than the SFMS. Blue triangles represent field starbursts with individual CO observations (see Methods). The faint red points show massive $\left(M_{*}>10^{10} M_{\odot}\right)$ rotation-dominated SBs at $z>4$ recently found $^{18-21}$, but no information on their environment is yet available. The light-blue area shows $V_{\max } / \sigma_{0}$ values for star-forming galaxies from Illustris-TNG50 simulations in the mass range $10^{9}-10^{11} M_{\odot}{ }^{16}$. The two lines with the light green and pink shaded regions describe $V_{\max } / \sigma_{0}$ as a function of the gas fraction $\left(f_{\text {gas }}\right)$ and disk instability $(Q)^{15}$, where a $=\sqrt{2}$ for a disk with constant rotational velocity. According to this model, the rotational support of a gas disk $V_{\max } / \sigma_{0}$ increases with the stellar mass and decreases with the redshift. The two cluster SBs have rotation-dominated gas disks with $V_{\max } / \sigma_{0}$ values more than three times larger than field SBs and most literature samples at a similar redshift. Right: The Toomre parameter $Q$ (see Methods for calculation) as a function of the main-sequence offset. The solid grey line and shaded area highlight the $\mathrm{SFMS}^{9}$ position and the $\pm 3 \times \Delta \mathrm{MS}$ region, commonly used to separate MS from SB galaxies. The $Q$ values calculated using the $3.2 \mathrm{~mm}$ dust continuum, the $\delta_{\mathrm{GDR}}$, and the CO-based gas masses with the metallicity-dependent conversion factors $\left(\alpha_{\mathrm{CO}}(Z) \sim 4\right)$ are shown by opened, filled light, and filled dark stars, respectively. The blue dashed line indicates the threshold $Q_{\text {crit }}=0.67$ below which a thick disk (as assumed in our gas kinematic model) becomes gravitationally unstable. This suggests that our two SBs are gravitationally unstable gas disks.

use the gas mass obtained both from dust continuum emission at $3.2 \mathrm{~mm}$ and the $\mathrm{CO}(1-0)$ luminosity (see Methods for the calculation). For the four $\mathrm{SBs} / \mathrm{MSs}$, the derived $Q$ values decrease as the SFR offset to the SFMS increases (right panel of Fig. 3). The two SBs present $Q$ values much smaller than $Q_{\text {crit }}$. We note that using the local starbursts conversion factor $\left(\alpha_{\mathrm{CO}, \mathrm{SB}}=0.8 M_{\odot}\left(\mathrm{K} \mathrm{km} \mathrm{s}^{-1} \mathrm{pc}^{2}\right)^{-1}\right)$ for the two SBs, the derived Toomre parameter $Q$ values will increase by a factor of five but still remain below $Q_{\text {crit }}$. The low Toomre $Q$ means that the gas disk can easily collapse due to gravitational instabilities, leading to an extreme starburst. Thus, the most likely triggering mechanism of these two SBs is the gravitational instability of their gas disks. In the field, recent work also found evidence of efficient star formation induced by gravitational instability in a $z=4.3$ starburst galaxy ${ }^{23}$.

To summarize, the dynamically cold, rotating gas disks of the two SBs are mainly due to low gas turbulent motions. Their intrinsic velocity dispersions are extremely low, with $\sigma_{0} \sim 20-30 \mathrm{~km} \mathrm{~s}^{-1}$, while the two MSs have $\sigma_{0} \sim 90-110$ $\mathrm{km} \mathrm{s}^{-1}$ (see Extended Data Table 3). Their $\sigma_{0}$ values are about three times lower than that of field SBs at similar redshiftsbased on molecular gas observations from 2D/3D kinematic modelling (blue triangles in Fig. 4, see Methods), and even field MS galaxies. We note that the $\sigma_{0}$ values of the two cluster SBs are consistent with those of a group of massive $\left(M_{*}>10^{10} M_{\odot}\right)$ rotation-dominated SBs recently found ${ }^{18-21}$ at $z>4$ (faint red points in Fig. 4). In general, the gas in field SBs at $z=2-3$ (blue and orange markers in Fig. 4 , see Methods) seems slightly more turbulent than that in field MSs, with a higher median $\sigma_{0}$ by a factor of $\sim 1.5$. Hence, the two cluster SBs exhibit strikingly different kinematic properties compared to field SBs at $z \sim 2.5$, whether observed by molecular gas or ionized gas. These results challenge our current understanding of the physical origin of high- $z$ starbursts in clusters and hint that mergers and interactions are not the only way to trigger starbursts. The high gas fractions ( 0.7; see Extended Data Table 2) and the suppressed ve- 


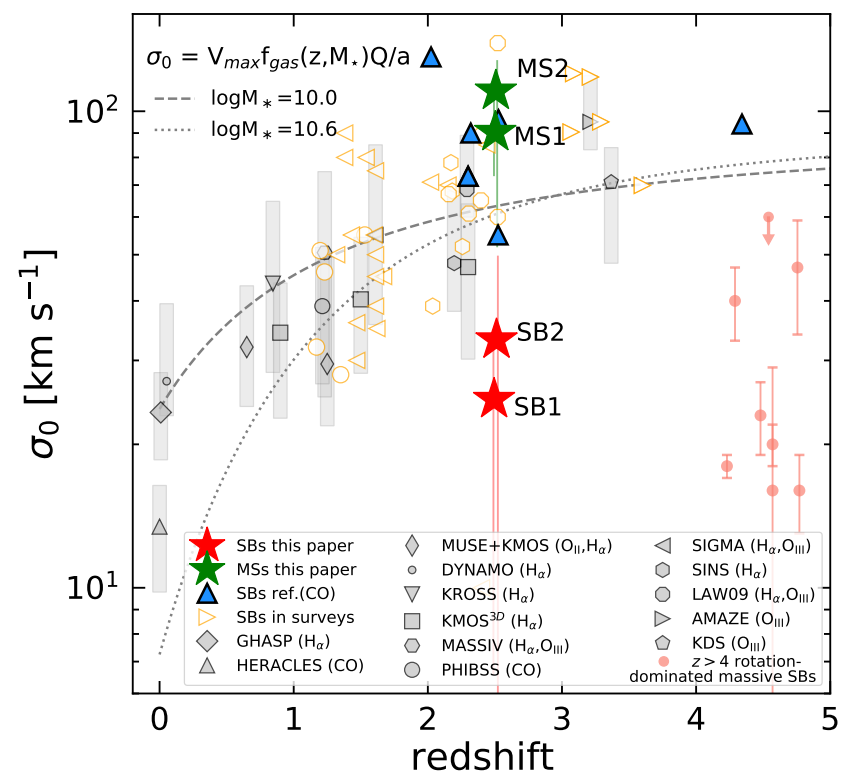

Figure 4: Comparison of gas turbulence between the four cluster SBs/MSs and field galaxies. The intrinsic velocity dispersion increases with redshift. The symbol convention and the two lines are the same as in the left panel of Fig. 3 The two lines show the relation between the gas velocity dispersion, the gas fraction, and the disk instability for different stellar masses ${ }^{15}$. Here we assume $V_{\max }=130 \mathrm{~km} \mathrm{~s}^{-1}, \mathrm{Q}=1$, and $\mathrm{a}=\sqrt{2}$ for a disk with constant rotational velocity. These tracks indicate that while $\sigma_{0}$ predictions from this relation depend on $M_{*}$, this dependency mostly vanishes at $z>2$. The two cluster SBs have lower $\sigma_{0}$ than field SBs as well as most literature samples. Their uncertainties are derived from our simulation (see Methods).

locity dispersions of these two SBs yield highly unstable gas disks, which in turn induce efficient starburst activities (Fig. 3).

We further explore the physical origin of the extremely low velocity dispersions and high gas fractions of the cluster SBs. The two primary energy sources of gas turbulence are stellar feedback and gravitational instability ${ }^{24,25}$; while high gas fraction could either be due to a merger or gas infall (cold gas accretion). The two SBs favour a scenario in which cold gas is accreted via co-rotating and co-planar streams ${ }^{e . g .26,27}$. Indeed, in such scenario most of the gravitational energy is converted to rotation rather than dispersion, building up angular momentum ${ }^{28,29}$. As a result, the two cluster SBs would have high gas fractions, low turbulent gas disks with the high rotational velocities $\left(V_{\max } \sim 400-500 \mathrm{~km} \mathrm{~s}^{-1}\right.$; see Extended Data Table 3), relatively extended gas disk sizes, and low $\mathrm{CO}$ excitation $R_{31}$ similar to that of $z=2-3 \mathrm{MS}$ galaxies (see Methods). Simulations do predict a high occurrence rate of such configurations of co-rotating and co-planar cold gas accretion in massive galaxies/halos ${ }^{29,30}$, for which galaxy merger rate is low compared to the orbital time. More specifically, the cold gas disk with $V_{\max } / \sigma_{0} \simeq 5$ in a $z \sim 3.5$ galaxy, with a stellar mass of $M_{*} \sim 10^{10} M_{\odot}$, typically survives for $\sim 5$ orbital periods (a duration time of $\sim 410 \mathrm{Myr})^{29}$, before being disrupted by mergers. Assuming the same duration time of 410 Myr between two merger events, we infer that our two cluster SBs, which have more massive cold gas disks with larger $V_{\max } / \sigma_{0}>10$, would survive for $\gtrsim 15$ orbital periods. In summary, the unique properties of the two cluster SBs (high gas fraction and suppressed velocity dispersion) support the scenario of co-rotating and co-planar cold gas accretion, as the cause of their highly efficient star formation. This may represent an important avenue other than mergers/interactions in triggering starbursts at high redshifts, at least in massive halos. Characterizing the environment of the recently found massive $\left(M_{*}>10^{10} M_{\odot}\right)$ rotation-dominated disks at $z>4$ with suppressed velocity dispersion presents an unique opportunity to support further this scenario.

1. Dressler, A. Galaxy morphology in rich clusters: implications for the formation and evolution of galaxies. Astrophys. J. 236, 351-365 (1980).

2. Thomas, D. et al. The Epochs of Early-Type Galaxy Formation as a Function of Environment. Astrophys. J. 621, 673 (2005).

3. Finlator, K. et al. The Physical and Photometric Properties of High-Redshift Galaxies in Cosmological Hydrodynamic Simulations. Astrophys. J. 639, 672-694 (2006).

4. Casey, C. M. The Ubiquity of Coeval Starbursts in Massive Galaxy Cluster Progenitors. Astrophys. J. 824, 36 (2016).

5. Miller, T. B. et al. A massive core for a cluster of galaxies at a redshift of 4.3. Nature 556, 469-472 (2018).

6. Oteo, I. et al. An Extreme Protocluster of Luminous Dusty Starbursts in the Early Universe. Astrophys. J. 856, 72 (2018).

7. Kravtsov, A. V. \& Borgani, S. Formation of Galaxy Clusters. Annu. Rev. Astron. Astrophys. 50, 353-409 (2012).

8. Wang, T. et al. Discovery of a Galaxy Cluster with a Violently Starbursting Core at $\mathrm{z}=2.506$. Astrophys. J. 828, 56 (2016).

9. Schreiber, C. et al. The Herschel view of the dominant mode of galaxy growth from $\mathrm{z}=4$ to the present day. Astron. Astrophys. 575, A74 (2015).

10. Wang, T. et al. Revealing the Environmental Dependence of Molecular Gas Content in a Distant X-Ray Cluster at z $=2.51$. Astrophys. J. Let. 867, L29 (2018).

11. Tacconi, L. J. et al. PHIBSS: Unified Scaling Relations of Gas Depletion Time and Molecular Gas Fractions. Astrophys. J. 853, 179 (2018).

12. Liu, D. et al. Automated Mining of the ALMA Archive in the COSMOS Field $(\mathrm{A}<\mathrm{SUP}>3</$ SUP $>$ COSMOS $)$. II. Cold Molecular Gas Evolution out to Redshift 6. Astrophys. J. 887, 235 (2019). 
13. Scoville, N. et al. Evolution of Interstellar Medium, Star Formation, and Accretion at High Redshift. Astrophys. J. 837, 150 (2017).

14. Bouché, N. et al. GalPak ${ }^{3 D}$ : A Bayesian Parametric Tool for Extracting Morphokinematics of Galaxies from 3D Data. Astron. J. 150, 92 (2015).

15. Wisnioski, E. et al. The KMOS ${ }^{3 D}$ Survey: Design, First Results, and the Evolution of Galaxy Kinematics from $0.7<=$ $\mathrm{z}<=2.7$. Astrophys. J. 799, 209-236 (2015).

16. Pillepich, A. et al. First results from the TNG50 simulation: the evolution of stellar and gaseous discs across cosmic time. Mon. Not. R. Astron. Soc. 490, 3196-3233 (2019).

17. Förster Schreiber, N. M. \& Wuyts, S. Star-Forming Galaxies at Cosmic Noon. Annu. Rev. Astron. Astrophys. 58, 661-725 (2020).

18. Rizzo, F. et al. A dynamically cold disk galaxy in the early Universe. Nature 584, 201-204 (2020).

19. Rizzo, F. et al. Dynamical properties of z 4.5 dusty starforming galaxies and their connection with local early-type galaxies. Mon. Not. R. Astron. Soc. 507, 3952 (2021).

20. Lelli, F. et al. A massive stellar bulge in a regularly rotating galaxy 1.2 billion years after the Big Bang. Science 371, 713$716(2021)$.

21. Fraternali, F. et al. Fast rotating and low-turbulence discs at $\mathrm{z} \simeq$ 4.5: Dynamical evidence of their evolution into local early-type galaxies. Astron. Astrophys. 647, A194 (2021).

22. Toomre, A. On the gravitational stability of a disk of stars. Astrophys. J. 139, 1217-1238 (1964).

23. Tadaki, K. et al. The gravitationally unstable gas disk of a starburst galaxy 12 billion years ago. Nature 560, 613-616 (2018).

24. Krumholz, M. R. \& Burkhart, B. Is turbulence in the interstellar medium driven by feedback or gravity? An observational test. Mon. Not. R. Astron. Soc. 458, 1671-1677 (2016).

25. Krumholz, M. R. et al. A unified model for galactic discs: star formation, turbulence driving, and mass transport. Mon. Not. R. Astron. Soc. 477, 2716 (2018).

26. Danovich, M. et al. Coplanar streams, pancakes and angularmomentum exchange in high-z disc galaxies. Mon. Not. R. Astron. Soc. 422, 1732 (2012).

27. Danovich, M. et al. Four phases of angular-momentum buildup in high-z galaxies: from cosmic-web streams through an extended ring to disc and bulge. Mon. Not. R. Astron. Soc. 449, 2087 (2015).

28. Kretschmer, M. et al. Rapid filamentary accretion as the origin of extended thin discs. Mon. Not. R. Astron. Soc. 497, 4346 (2020).

29. Kretschmer, M. et al. Evaluating galaxy dynamical masses from kinematics and jeans equilibrium in simulations. Mon. Not. R. Astron. Soc. 503, 5238-5253 (2021).
30. Dekel, A. et al. A mass threshold for galactic gas discs by spin flips. Mon. Not. R. Astron. Soc. 493, 4126 (2020). 


\section{Methods}

Cosmology. We assume cosmological parameters of $H_{0}=70$ $\mathrm{km} \mathrm{s}^{-1} \mathrm{Mpc}^{-1}, \Omega_{\mathrm{M}}=0.3$, and $\Omega_{\Lambda}=0.7$ and adopt a Chabrier initial mass function (IMF) ${ }^{31}$ to estimate star formation rate (SFR). When necessary, data from the literature has been converted with conversion factor of SFR (Salpeter IMF) $)^{32}=$ $1.7 \times$ SFR $(\text { Chabrier IMF })^{31}$ and $\mathrm{M}_{*}($ Salpeter IMF $)=1.7 \times$ $\mathrm{M}_{*}$ (Chabrier IMF).

Observations. We carried out observations of the $\mathrm{CO}(3-2)$ transitions at the rest-frame frequency of $345.796 \mathrm{GHz}$ ( $98.62977 \mathrm{GHz}$ in the observed frame) for the galaxy cluster CLJ1001 ${ }^{8}$ (see also ${ }^{10,33-37}$ ) at $z=2.51$ with ALMA band-3 receivers. These ALMA Cycle 4 observations (2016.1.01155.S; PI: Tao Wang) were taken between 2016 November and 2017 August with a total observing time, including calibration and overheads, approximately 7 hours. On-source time is 1.6 hours and 2.2 hours with the C40-4 and C40-7 array configurations, respectively. The observations have been done with different array configurations to obtain reliable measurements of total flux and resolved kinematics information.

The calibration was performed using version 5.3.0 of the Common Astronomy Software Application package $(\mathrm{CASA})^{38}$ with a standard pipeline. We carried out the data reduction in two cases. The case 1 is to obtain a highresolution cube of $\mathrm{CO}(3-2)$ line, we first combined two configurations (C40-4 and C40-7) data to form a single visibility table (UV table), using visibility weights proportional to 1:4. Imaging was carried out using the tclean task with $0.04^{\prime \prime}$ pixels and a channel width of $30 \mathrm{~km} \mathrm{~s}^{-1}$ with a briggs weighting of robust $=0.5$ scheme. The resulting data cube has synthesized beam size of $0.31^{\prime \prime} \times 0.25^{\prime \prime}(\sim 2.48 \mathrm{kpc} \times 2.00 \mathrm{kpc}$ in physical scale) with the rms of $\sim 110-120 \mu \mathrm{Jy}$ beam $^{-1}$ per channel at the phase center. The case 2 is to derive the total flux of our sources, we combined two configurations data with visibility weights proportional to $1: 1$. We used $0.2^{\prime \prime}$ pixels and a channel width of $90 \mathrm{~km} \mathrm{~s}^{-1}$ with uvtaper of $1^{\prime \prime}$ and a natural weighting scheme for imaging. The natural weighting provides a better sensitivity, enabling us to measure the total flux more robustly. The resulting data cube has synthesized beam size of $1.54^{\prime \prime} \times 1.37^{\prime \prime}(\sim 12.32 \mathrm{kpc}$ $\times 10.96 \mathrm{kpc}$ in physical scale) and the central rms level of $\sim 60 \mu \mathrm{Jy}$ beam $^{-1}$ per channel.

We also created the observed $3.2 \mathrm{~mm}$ continuum maps using briggs weighting (robust $=0.5$ ) after excluding the frequency range of the $\mathrm{CO}(3-2)$ line. We used the same method as for the $\mathrm{CO}(3-2)$ cubes to create two continuum maps with different angular resolutions. The rms level is $\sim 6.9 \mu \mathrm{Jy} \mathrm{beam}^{-1}$ in the map of $0.30^{\prime \prime} \times 0.24^{\prime \prime}$ spatial resolution $(\sim 2.40 \mathrm{kpc} \times 1.92 \mathrm{kpc}$ in physical scale $)$ and $\sim 4.3 \mu \mathrm{Jy}$ beam $^{-1}$ in the map of $1.12^{\prime \prime} \times 1.06^{\prime \prime}$ spatial reso- lution $(\sim 8.96 \mathrm{kpc} \times 8.48 \mathrm{kpc}$ in physical scale $)$, respectively.

Starburst and main-sequence galaxies. We focus on the four massive member galaxies with $\log \left(M_{*} / M_{\odot}\right)>10.8$ from the galaxy cluster CLJ1001 at $z=2.51$. All four galaxies are located in the central region of the ALMA primary beam (a full beam width at half power of 53") with the highest signal-to-noise ratio $(S / N \gg 10)$ among all $\mathrm{CO}(3-2)$ detections (Fig. 11, allowing a detailed study of their kinematics. Stellar mass and total infrared luminosity used to calculate $\mathrm{SFR}^{39}$, which were derived from the spectral energy distribution (SED) fitting ${ }^{8,10}$, are presented in Extended Data Table 1. Two cluster members are classical SBs, i.e., located $0.7 \mathrm{dex}$ and 0.5 dex above the SFMS ${ }^{9}$. Two other members are identified as MSs, as being located 0.1 dex and -0.4 dex from the SFMS. According to their distances from the SFMS ( $\triangle \mathrm{MS})$, the four galaxies are then named SB1, SB2, MS1, and MS2. We note that the MS1 consumes molecular gas (estimated based on the $\mathrm{CO}(1-0)$ emission line) as fast as two SBs, with the gas depletion time $t_{\text {dep }} \sim 200 \mathrm{Myr}$ (Extended Data Table 2). The typical $t_{\text {dep }}$ of MS galaxies with a stellar mass of $10^{11} M_{\odot}$ at $z=2.5$ is $\sim 600 \mathrm{Myr}^{11}$. Therefore, the MS1 is likely consistent with the discovery of starbursts hidden in the MS, which were thought to be in the last stage of star formation quenching before passivization ${ }^{40}$.

Structural properties of $\mathbf{C O}(3-2)$. We measure the structural properties of the four cluster members. To avoid uncertainties from the imaging process, we derive the source sizes with the visibility $\mathrm{CO}(3-2)$ data in the $U V$ plane by fitting an elliptical Gaussian (task uvmodel fit). The best-fit values of radius are $\mathrm{r}_{\mathrm{CO}}=0.5 \times \mathrm{FWHM}=2.05$ $\pm 0.10 \mathrm{kpc}, 1.42 \pm 0.11 \mathrm{kpc}, 1.77 \pm 0.20 \mathrm{kpc}$ and $0.79 \pm$ $0.20 \mathrm{kpc}$ for SB1, SB2, MS1 and MS2, respectively. We also perform the two-dimensional elliptical Gaussian fitting on the high-resolution Moment 0 map. The deconvolved half FWHM values are $1.72 \pm 0.22 \mathrm{kpc}, 1.55 \pm 0.26 \mathrm{kpc}$, $2.24 \pm 0.33 \mathrm{kpc}$, and $1.85 \pm 0.37 \mathrm{kpc}$, respectively. The two $\mathrm{SBs}$ with higher $\mathrm{S} / \mathrm{N}$ than the two MSs show intrinsic sizes consistent in both methods within the error. As a reference, assuming a lack of significant size variation between the $\mathrm{CO}$ and dust continuum ${ }^{\text {e.g.41 }}$, the typical dust size for galaxies at $z=2.5$ with stellar masses of $10^{11} M_{\odot}$ is $\sim 1 \mathrm{kpc}$ in radius from GOODS-ALMA $1.1 \mathrm{~mm}$ survey ${ }^{42}$. The two SBs do not show compact disks that meet the sub-millimetre compactness criteria ${ }^{43}$.

CO excitation. The $\mathrm{CO}(3-2)$ line flux is measured from a two-dimensional (2D) Gaussian fitting on the velocity-integrated intensity map (Moment 0 map) with the velocity range shown in Fig. 1. Then we calculate its luminosity $L_{\mathrm{CO}(3-2)}^{\prime}{ }^{44}$. The $\mathrm{CO}(1-0)$ line luminosity $L_{\mathrm{CO}(1-0)}^{\prime}$ has already been obtained with the Karl G. Jansky Very Large Array (JVLA) observations ${ }^{10}$. Therefore, we 
can derive the $\mathrm{CO}$ excitation $R_{31}$ with the luminosity ratio $L_{\mathrm{CO}(3-2)}^{\prime} / L_{\mathrm{CO}(1-0)}^{\prime}$. The values are listed in Extended Data Table 1 .

Dust mass. To obtain dust mass, $M_{\text {dust }}$, we perform the FIR SED fitting with CIGALE ${ }^{1}$ Code Investigating Galaxies Emission) ${ }^{45,46}$. We fit data from $24 \mu \mathrm{m}$ up to the millimeter wavelengths (see the data at $24 \mu \mathrm{m}, 100$ $\mu \mathrm{m}, 160 \mu \mathrm{m}, 870 \mu \mathrm{m}, 1.8 \mathrm{~mm}$ in $\mathrm{W} 16^{8}$, and the data at $3.2 \mathrm{~mm}$ in Extended Data Table 1). The dust infrared emission model ${ }^{47}$ has an extensive grid of parameters: the mass fraction of the polycyclic aromatic hydrocarbon (PAH) of $\mathrm{q}_{\mathrm{PAH}}=0.47-7.32 \%$, the minimum radiation field of $U_{\min }=0.1-50$, the spectral index of $\alpha=1-3$, the fraction illuminated from $U_{\min }$ to $U_{\max }$ of $\gamma=0.0001-1$. Note that we do not find a sign of a significant contribution of an AGN in the FIR SED of our four SBs/MSs, consistent with previous literature constraints ${ }^{8,10}$. The derived $M_{\text {dust }}$ is given in Extended Data Table 1 .

Molecular gas mass. We adopt three commonly used methods to obtain the total molecular gas mass, $M_{\text {gas }}$, based on: (i) the CO line, (ii) the gas-to-dust ratio, and (iii) the Rayleigh-Jeans (RJ) tail dust continuum (see Extended Data Table 2).

To obtain the total molecular gas mass $M_{\text {gas,CO, we use }}$ $\mathrm{CO}(1-0)$ luminosity ${ }^{10}$ instead of $\mathrm{CO}(3-2)$ luminosity to avoid uncertainties caused by the $\mathrm{CO}$ excitation ladder. The $M_{\mathrm{gas}, \mathrm{CO}}$ is calculated by $M_{\mathrm{gas}, \mathrm{CO}}=\alpha_{\mathrm{CO}}(Z) L_{\mathrm{CO}(1-0)}^{\prime}$, including a factor 1.36 correction for helium. As was done in our previous work $^{10}$, the metallicity-dependent $\mathrm{CO}-$ to- $\mathrm{H}_{2}$ conversion factor, $\alpha_{\mathrm{CO}}(Z)$, is determined from the massmetallicity relation ${ }^{11,48}$.

The gas mass, $M_{\text {gas,GDR, }}$ can also be determined through $M_{\text {dust }}$ by employing the gas-to-dust ratio with a metallicity dependency ${ }^{49}$. We used $M_{\text {gas }}=\delta_{\mathrm{GDR}} M_{\text {dust }}$ and $\log \delta_{\mathrm{GDR}}=$ $10.54-0.99(12+\log (\mathrm{O} / \mathrm{H}))$. The metallicity is determined from the redshift-dependent mass-metallicity relation ${ }^{48}: 12$ $+\log (\mathrm{O} / \mathrm{H})=\mathrm{a}-0.087\left(\log M_{*}-b\right)^{2}$, where $\mathrm{a}=8.74$ and $\mathrm{b}=10.4+4.46 \log (1+z)-1.78 \log (1+z)^{2}$. We adopted an uncertainty of 0.15 dex in the metallicities ${ }^{49}$.

The long-wavelength RJ-tail $(\geq \sim 200 \mu \mathrm{m}$ in the restframe) of dust continuum emission is nearly always optically thin, providing a reliable probe of the total dust content. The RJ-tail method ${ }^{50}$ is based on an empirical calibration between the molecular gas content and the dust continuum at the rest-frame $850 \mu \mathrm{m}$, which was obtained after considering a sample of low-redshift star-forming galaxies, ultra-luminous infrared galaxies, and $z=2-3$ submillimeter galaxies. We use our single ALMA $3.2 \mathrm{~mm}$ measurement at the RJ-tail (corrected using published erratum $)^{50}$ to derive total molec-

\footnotetext{
${ }^{1}$ CIGALE: https://cigale.lam.fr
}

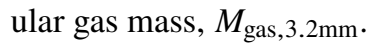

For our four SBs/MSs, the $M_{\mathrm{gas}, \mathrm{CO}}, M_{\mathrm{gas}, \mathrm{GDR}}$, and $M_{\text {gas, } 3.2 \mathrm{~mm}}$ derived from three different methods are consistent with each other (see Extended Data Table 2).

As a discussion, we note that the derived $\alpha_{\mathrm{CO}}(Z)^{10}$ is close to the Milky Way (MW) value (see Extended Data Table 2). In general, two conversion factors are commonly used in the literature to convert $\mathrm{CO}$ luminosities into gas masses, the Milky Way $\left(\alpha_{\mathrm{CO}, \mathrm{MW}}=4.36 M_{\odot}\left(\mathrm{K} \mathrm{km} \mathrm{s}^{-1}\right.\right.$ $\left.\left.\mathrm{pc}^{2}\right)^{-1}\right)$ and local starbursts $\left(\alpha_{\mathrm{CO}, \mathrm{SB}}=0.8 M_{\odot}\left(\mathrm{K} \mathrm{km} \mathrm{s}^{-1}\right.\right.$ $\left.\mathrm{pc}^{2}\right)^{-151,52}$ ) values. However, 1) it has been found that distant starburst galaxies can exhibit conversion factors typical of the Milky Way, when they present extended instead of compact star formation ${ }^{\text {e.g. } 43}$ and/or large gas fractions. Here the two SBs exhibit both relatively extended $\mathrm{CO}$ sizes and large gas fractions (from the dust continuum), favouring a MW-like $\alpha_{\mathrm{CO}} ; 2$ ) starburst galaxies generally have higher $\mathrm{CO}$ excitation rates relative to main-sequence objects ${ }^{\text {e.g.43,53. }}$ Here the $R_{31} \sim 0.8$ of the two SBs is similar to that of $z=2-3 \mathrm{MS}$ galaxies (the median $R_{31}$ is $0.84 \pm 0.26$ ) from the VLASPECS survey ${ }^{54}$, implying that they have MW-like ISM conditions; 3) using a local SB conversion factor will result in very low gas-to-dust ratios (GDR 20), making the two SBs extreme cases. The result GDRs are 4-5 times lower than the typical GDR of solar-metallicity galaxies regardless of redshift ${ }^{55}$, and $>2$ times lower than GN20 ${ }^{49}$. Hence, all these evidences are consistent with our derived $\alpha_{\mathrm{CO}}(Z)$. We will however show that even if we had chosen a local SB conversion factor for the two SBs, the main conclusions of the paper would remain unchanged.

Disk modelling and gas kinematics. We fit the $\mathrm{CO}(3-2)$ line cubes using the GalPAK ${ }^{3 D}$ code $^{14}$. $\operatorname{GalPAK}^{3 D}$ is a Bayesian parametric tool, using a Markov Chain Monte Carlo (MCMC) approach to derive the intrinsic galaxy parameters and kinematic properties from a 3D data cube. The model is convolved with a three-dimensional kernel to account for the instrument point spread function (PSF) and the line spread function (LSF) smearing effect. The GalPAK $^{3 D}$ can thus return the intrinsic galaxy properties. We adopt a thick exponential-disk light profile and an arctan rotational velocity curve to fit the $0.3^{\prime \prime}$-resolution cube so as to determine the maximum circular velocity $V_{\max }$ and intrinsic velocity dispersion $\sigma_{0}$. The arctan rotational velocity curve is $V_{\text {rot }} \propto V_{\max } \arctan \left(r / r_{\mathrm{v}}\right)$, where $r_{\mathrm{V}}$ is the turnover radius. Fig. 2 shows the best-fit models and residuals, based on the last $30 \%$ chain fraction with 20,000 iterations. For the four SBs/MSs, our gas kinematic modelling provides a good description of the observed Moment 0, 1, and 2 maps (Extended Data Fig. 3, 4, 5, and 6. We also plot the full MCMC chain from GalPAK ${ }^{3 D}$ for the two SBs and two MSs with 20,000 iterations to confirm the convergence of rotational velocity and intrinsic velocity 
dispersion (Extended Data Fig. 11). The MCMC results of the fitting procedure are given in Extended Data Fig. 7, 8 , 9 , and 10, showing the posterior probability distributions of the model parameters and their marginalized distributions. The best-fitting values and their $1 \sigma$ uncertainties are summarized in Extended Data Table 3

To investigate the performance of the fit, we compare the rotation velocity and velocity dispersion profiles from the observed data with that from the best-fit PSF-convolved model data. All values are derived from 1D spectra extracted using circular apertures with diameters equal to angular resolution $0.31^{\prime \prime}$ along the kinematic major axis. As shown in Extended Data Fig. 2, the profiles from dynamical models and observations are consistent. Some points deviate from the models because of the flux contamination of neighbouring sources. The derived best-fit $\sigma_{0}$ from GalPAK ${ }^{3 D}$ is lower than the lowest observed velocity dispersion, which is reasonable because the latter is still affected by beam smearing effect even at the large radii probed by our observations.

Noticing that the best fit $\sigma_{0}$ of SB1 is lower than the $\mathrm{CO}(3-2)$ channel width of $30 \mathrm{~km} \mathrm{~s}^{-1}$, we further test the robustness of our fits. We apply the same analysis to the data cubes under the original channel width of $\sim 12 \mathrm{~km} \mathrm{~s}^{-1}$. The results are consistent with each other within errors. Therefore, considering that better sensitivity can help detect fainter galaxy edges and better sample the flat part of the rotation curves, we have adopted the values from the data cubes with a channel width of $30 \mathrm{~km} \mathrm{~s}^{-1}$ in this work.

Finally, we make a simple simulation to test the robustness of $\sigma_{0}$ and $V_{\max }$ given by the GalPAK ${ }^{3 D}$. Our purpose is to check 1) whether there is a global offset of the derived values or not; 2) the reliability of the output uncertainties. To start, we randomly inject the best-fit PSF-convolved model data cube into the observed data cube (at the same positions as our galaxies but at a different velocity/frequency to avoid the contamination of the $\mathrm{CO}$ emission from real sources). Then, we run the $\operatorname{GalPAK}^{3 D}$ to derive $\sigma_{0}$ and $V_{\max }$. These two steps are repeated 100 times for each galaxy. In the end, we have distributions of $\sigma_{0}$ and $V_{\max }$. We calculate the median $\sigma_{0}$ and uncertainty (16-84th percentile range) values for the $\mathrm{SB} 1, \mathrm{SB} 2, \mathrm{MS} 1$, and MS2, respectively. In general, there is no global offset of the derived values from the GalPAK ${ }^{3 D}$, but the uncertainty is greatly underestimated by a factor of 5. Thus, we use the uncertainties of $\sigma_{0}$ and $V_{\max }$ given by this simulation in the main body of this paper, Fig. 3, 4, and Extended Data Table 3

Our SBs/MSs are located in a dense environment. To better understand the environmental effect on gas turbulence, we compare the four cluster members with field galaxies from the literature. Compared to the field star-forming samples, the two SBs have significantly higher $V_{\max } / \sigma_{0}$ and lower $\sigma_{0}$ values at similar redshifts, especially compared to the field starbursts. The data included in Fig. 3 and Fig. 4 contains molecular and ionized gas observations. The ionized gas samples, sorted by redshifts from the lowest to the highest, are from surveys GHASP $\left(\log M_{*}^{a v g}\left[M_{\odot}\right]=10.6\right)^{56}$, DYNAMO $\left(\log M_{*}^{a v g}\left[M_{\odot}\right]=10.3\right)^{57}, \quad$ MUSE $\quad\left(\log M_{*}^{a v g}\left[M_{\odot}\right]=9.4\right.$, 9.8 at $z \sim 0.7,1.3)^{58}, \operatorname{KROSS}\left(\log M_{*}^{a v g}\left[M_{\odot}\right]=9.9\right)^{59}$, $\mathrm{KMOS}^{3 D}\left(\log M_{*}^{a v g}\left[M_{\odot}\right]=10.5,10.6,10.7\right.$ at $z \sim 0.9$, $1.5,2.3)^{15,60}$, MASSIV $\left(\log M_{*}^{a v g}\left[M_{\odot}\right]=10.2\right)^{61}$, SIGMA $\left(\log M_{*}^{a v g}\left[M_{\odot}\right]=10.0\right)^{62}, \quad$ SINS $\left(\log M_{*}^{a v g}\left[M_{\odot}\right]=10.6\right)^{63,64}$, LAW09 $\quad\left(\log M_{*}^{a v g}\left[M_{\odot}\right]=10.3\right)^{65}, \quad$ AMAZE $\left(\log M_{*}^{a v g}\left[M_{\odot}\right]=9.9\right)^{66}$, and $\operatorname{KDS}\left(\log M_{*}^{a v g}\left[M_{\odot}\right]=9.8\right)^{67}$. The molecular gas samples, sorted by redshifts, are from the HERACLES survey $\left(\log M_{*}^{a v g}\left[M_{\odot}\right]=10.5\right)^{68,69}$, PHIBSS survey $\left(\log M_{*}^{a v g}\left[M_{\odot}\right]=11.0\right)^{70}$. Some field SBs with individual $\mathrm{CO}$ observations ${ }^{71-75}$ are in blue triangles in Fig. 4. We note that although the velocity dispersion measured from the molecular gas is $\sim 10-15 \mathrm{~km} \mathrm{~s}^{-1}$ lower than the ionized gas (with extra contributions from thermal broadening and expansion of the HII regions) in the local Universe, this difference becomes smaller with increasing redshift ${ }^{60}$. When possible, we also identified the field starbursts (orange symbols in Fig. 4) within the above star-forming samples, requiring their SFR to be at least 0.5 dex higher than the MS. In general, the gas in these field starbursts is slightly more turbulent than in main-sequence galaxies, showing a higher $\sigma_{0}$, especially at $2<z<4$.

Toomre $\mathbf{Q}$ parameter. A rotating, symmetric gas disk is unstable to the gravitational fragmentation if the Toomre $Q$ parameter, $Q=\kappa \sigma_{0 \text {,gas }} /\left(\pi G \Sigma_{\text {gas }}\right)^{22}$, is below a threshold value $Q_{\text {crit }}$. The $Q_{\text {crit }}=1$ is for a thin gas disk, and $Q_{\text {crit }}=0.67$ for a thick gas disk. We calculate the gas surface density, $\Sigma_{\text {gas }}=0.5 M_{\text {gas }} /\left(\pi r_{1 / 2}^{2}\right)$, where $M_{\text {gas }}$ is derived from the $\mathrm{CO}(1-0)$ luminosity and dust continuum emission at 3.2 $\mathrm{mm}$. Here we adopt the maximum circular velocity $V_{\max }$, intrinsic velocity dispersion $\sigma_{0}$, the disk half-light radius $r_{1 / 2}$, and flat rotation curve with the epicyclic frequency $\kappa=\sqrt{2} V_{\max } / r_{1 / 2}{ }^{76}$. All these values are derived from the gas kinematic modelling. Fig. 3 p shows the $Q$ values of the four $\mathrm{SBs} / \mathrm{MSs}$ based on $\mathrm{CO}$ and dust continuum emission as a function of the main-sequence offset.

31. Chabrier, G. Galactic Stellar and Substellar Initial Mass Function. Publ. Astron. Soc. Pac. 809, 763-795 (2003).

32. Salpeter, E. The Luminosity Function and Stellar Evolution. Astrophys. J. 121, 161 (1955).

33. Casey, C. M. et al. A Massive, Distant Proto-cluster at $\mathrm{z}=2.47$ Caught in a Phase of Rapid Formation? Astrophys. J. 808, L33 (2015).

34. Daddi, E. et al. Radio Selection of the Most Distant Galaxy Clusters. Astrophys. J. 846, L31 (2017). 
35. Cucciati, O. et al. The progeny of a cosmic titan: a massive multi-component proto-supercluster in formation at $\mathrm{z}=2.45$ in VUDS. Astron. Astrophys. 619, A49 (2018).

36. Gómez-Guijarro, C. et al. Confirming Herschel Candidate Protoclusters from ALMA/VLA CO Observations. Astrophys. J. 872, 117 (2019).

37. Champagne, J. B. et al. Comprehensive Gas Characterization of $\mathrm{a} \mathrm{z}=2.5$ Protocluster: A Cluster Core Caught in the Beginning of Virialization? Astrophys. J. 913, 110 (2021).

38. McMullin, J. P. et al. CASA Architecture and Applications. In Shaw, R. A., Hill, F. \& Bell, D. J. (eds.) Astr. Soc. P., vol. 376 of Astronomical Society of the Pacific Conference Series, 127 (2007).

39. Kennicutt, R. C. \& Evans, N. J. Star Formation in the Milky Way and Nearby Galaxies. Annu. Rev. Astron. Astr. 50, 531608 (2012).

40. Elbaz, D. et al. Starbursts in and out of the star-formation main sequence. Astron. Astrophys. 616, A110 (2018).

41. Puglisi, A. et al. The Main Sequence at $\mathrm{z} \sim 1.3$ Contains a Sizable Fraction of Galaxies with Compact Star Formation Sizes: A New Population of Early Post-starbursts? Astrophys. J. 877, L23 (2019).

42. Gómez-Guijarro, C. et al. GOODS-ALMA 2.0: Source cata$\log$, number counts, and prevailing compact sizes in $1.1 \mathrm{~mm}$ galaxies. arXiv e-prints, arXiv:2106.13246 (2021).

43. Puglisi, A. et al. Sub-millimetre compactness as a critical dimension to understand the Main Sequence of star-forming galaxies. arXiv e-prints, arXiv:2103.12035 (2021).

44. Solomon, P. M. \& Vanden Bout, P. A. Molecular Gas at High Redshift. Annu. Rev. Astron. Astrophys. 43, 677-725 (2005).

45. Burgarella, D. et al. Star formation and dust attenuation properties in galaxies from a statistical ultraviolet-to-far-infrared analysis. Mon. Not. R. Astron. Soc. 360, 1413 (2005).

46. Noll, S. et al. Analysis of galaxy spectral energy distributions from far-UV to far-IR with CIGALE: studying a SINGS test sample. Astron. Astrophys. 507, 1793 (2009).

47. Draine, B. T. et al. Andromeda's Dust. Astrophys. J. 780, 172 (2014).

48. Genzel, R. et al. Combined CO and Dust Scaling Relations of Depletion Time and Molecular Gas Fractions with Cosmic Time, Specific Star-formation Rate, and Stellar Mass. Astrophys. J. 800, 20 (2015).

49. Magdis, G. E. et al. The Evolving Interstellar Medium of Star-forming Galaxies since $\mathrm{z}=2$ as Probed by Their Infrared Spectral Energy Distributions. Astrophys. J. 760, 6 (2012).

50. Scoville, N. et al. ISM Masses and the Star formation Law at $\mathrm{Z}=1$ to 6: ALMA Observations of Dust Continuum in 145 Galaxies in the COSMOS Survey Field. Astrophys. J. 820, 83-104 (2016).
51. Downes, D. \& Solomon, P. M. Rotating Nuclear Rings and Extreme Starbursts in Ultraluminous Galaxies. Astrophys. J. 507, 615-654 (1998).

52. Tacconi, L. J. et al. Submillimeter Galaxies at z 2: Evidence for Major Mergers and Constraints on Lifetimes, IMF, and CO- $\mathrm{H}_{2}$ Conversion Factor. Astrophys. J. 680, 246-262 (2008).

53. Valentino, F. et al. CO emission in distant galaxies on and above the main sequence. Astron. Astrophys. 641, A155 (2020).

54. Riechers, D. A. et al. VLA-ALMA Spectroscopic Survey in the Hubble Ultra Deep Field (VLASPECS): Total Cold Gas Masses and CO Line Ratios for $\mathrm{z}=2$-3 Main-sequence Galaxies. Astrophys. J. 896, L21 (2020).

55. Rémy-Ruyer, A. et al. Gas-to-dust mass ratios in local galaxies over a 2 dex metallicity range. Astron. Astrophys. 563, A31 (2014).

56. Epinat, B. et al. Evidence for strong dynamical evolution in disc galaxies through the last 11 Gyr. GHASP VIII - a local reference sample of rotating disc galaxies for high-redshift studies. Mon. Not. R. Astron. Soc. 401, 2113-2147 (2010).

57. Green, A. W. et al. DYNAMO - I. A sample of $\mathrm{H} \alpha$-luminous galaxies with resolved kinematics. Mon. Not. R. Astron. Soc. 437, 1070-1095 (2014).

58. Swinbank, A. M. et al. Angular momentum evolution of galaxies over the past $10 \mathrm{Gyr}$ : a MUSE and KMOS dynamical survey of 400 star-forming galaxies from $\mathrm{z}=0.3$ to 1.7. Mon. Not. $R$. Astron. Soc. 467, 3140-3159 (2017).

59. Johnson, H. L. et al. The KMOS Redshift One Spectroscopic Survey (KROSS): the origin of disc turbulence in $\mathrm{z} \approx 1$ starforming galaxies. Mon. Not. R. Astron. Soc. 474, 5076-5104 (2018).

60. Übler, H. et al. The Evolution and Origin of Ionized Gas Velocity Dispersion from $\mathrm{z} \sim 2.6$ to $\mathrm{z} \sim 0.6$ with $\mathrm{KMOS}^{3 D}$. Astrophys. J. 880, 48 (2019).

61. Epinat, B. et al. MASSIV: Mass Assembly Survey with SINFONI in VVDS. II. Kinematics and close environment classification. Astron. Astrophys. 539, A92 (2012).

62. Simons, R. C. et al. Kinematic Downsizing at $\mathrm{z} \sim 2$. Astrophys. J. 830, 14 (2016).

63. Förster Schreiber, N. M. et al. The SINS Survey: SINFONI Integral Field Spectroscopy of $\mathrm{z} \sim 2$ Star-forming Galaxies. Astrophys. J. 706, 1364-1428 (2009).

64. Cresci, G. et al. The SINS Survey: Modeling the Dynamics of $\mathrm{z} \sim 2$ Galaxies and the High-z Tully-Fisher Relation. Astrophys. J. 697, 115-132 (2009).

65. Law, D. R. et al. The Kiloparsec-scale Kinematics of Highredshift Star-forming Galaxies. Astrophys. J. 697, 2057-2082 (2009).

66. Gnerucci, A. et al. Dynamical properties of AMAZE and LSD galaxies from gas kinematics and the Tully-Fisher relation at $\mathrm{z}$ 3. Astron. Astrophys. 528, A88 (2011). 
67. Turner, O. J. et al. The KMOS Deep Survey (KDS) - I. Dynamical measurements of typical star-forming galaxies at $\mathrm{z} \simeq$ 3.5. Mon. Not. R. Astron. Soc. 471, 1280-1320 (2017).

68. Leroy, A. K. et al. The Star Formation Efficiency in Nearby Galaxies: Measuring Where Gas Forms Stars Effectively. Astron. J. 136, 2782-2845 (2008).

69. Leroy, A. K. et al. Heracles: The HERA CO Line Extragalactic Survey. Astron. J. 137, 4670-4696 (2009).

70. Tacconi, L. J. et al. Phibss: Molecular Gas Content and Scaling Relations in $\mathrm{z} \sim 1$-3 Massive, Main-sequence Star-forming Galaxies. Astrophys. J. 768, 74 (2013).

71. Calistro Rivera, G. et al. Resolving the ISM at the Peak of Cosmic Star Formation with ALMA: The Distribution of CO and Dust Continuum in $\mathrm{z} \sim 2.5$ Submillimeter Galaxies. Astrophys. J. 863, 56 (2018).

72. Barro, G. et al. Spatially Resolved Kinematics in the Central 1 $\mathrm{kpc}$ of a Compact Star-forming Galaxy at $\mathrm{z} \sim 2.3$ from ALMA CO Observations. Astrophys. J. Let. 851, L40 (2017).

73. Swinbank, A. M. et al. The Interstellar Medium in Distant Star-forming Galaxies: Turbulent Pressure, Fragmentation, and Cloud Scaling Relations in a Dense Gas Disk at $\mathrm{z}=2.3$. Astrophys. J. 742, 11 (2011).

74. Tadaki, K. et al. Rotating Starburst Cores in Massive Galaxies at $\mathrm{z}=2.5$. Astrophys. J. Let. 841, L25 (2017).

75. Tadaki, K. et al. CNO Emission of an Unlensed Submillimeter Galaxy at $\mathrm{z}=4.3$. Astrophys. J. 876, 1 (2019).

76. Binney, J. \& Tremaine, S. Galactic Dynamics: Second Edition. Princeton University Press, Princeton , 494-496 (2008).

77. Muzzin, A et al. A Public $\mathrm{K}_{s}$-selected Catalog in the COSMOS/ULTRAVISTA Field: Photometry, Photometric Redshifts, and Stellar Population Parameters. Astrophys. J. Supp. 206, 8 (2013).

Acknowledgements This paper makes use of the following ALMA data: ADS/JAO.ALMA \#2016.1.01155.S. ALMA is a partnership of ESO (representing its member states), NSF (USA), and NINS (Japan), together with NRC (Canada), NSC, ASIAA (Taiwan), and KASI (Republic of Korea), in cooperation with the Republic of Chile. The Joint ALMA Observatory is operated by ESO, AUI/NRAO, and NAOJ. This work is supported by the National Key Research and Development Program of China (No. 2017YFA0402703), and by the National Natural Science Foundation of China (Key Project No. 11733002 and Project No. 12173017). This work is also supported by the Programme National Cosmology et Galaxies (PNCG) of CNRS/INSU with INP and IN2P3, co-funded by CEA and CNES. M.-Y. X. acknowledges the support by China Scholarship Council (CSC). T.W. and K. K. acknowledges the support by NAOJ ALMA Scientific Research Grant Number 2017-06B. D. I. acknowledges the support by JSPS KAKENHI Grant Number JP21H01133.

Author Information The authors declare no competing interests.
Author Contributions T.W. conceived the work, proposed and carried out ALMA observations. M.-Y. X. and T. W. led the analysis and interpretation, and authored the majority of the text. M.-Y. X. carried out the ALMA data reductions with the help of X. L. and T.W., performed disk kinematic modelling and FIR SED fitting. D. E., D. I., L.-J. B., E. D., B. M., C. G.-G., S.-W. J., F. V., A. Z., R. G., S. M., G. B., Q.-S. G., K. K., and X.-L. Y. helped with various aspects of the analysis. M.-Y. X. and E. D. contributed to the simulation. All authors discussed the results and provided comments on the paper.

Data availability This study used the ALMA data 2016.1.01155.S, available at https://almascience.eso.org/aq/.

Code availability The ALMA data were reduced using the CASA pipeline version 5.3.0, available at https://casa.nrao.edu/casa_obtaining.shtml. The disk kinematic modelling code $\mathrm{GalPAK}^{3 D}$ is publicly available at http://galpak3d.univ-lyon1.fr and/or http://www.ascl.net/1501.014. 


\section{Extended Data}

Extended Data Table 1: Physical properties of the four SBs/MSs in CLJ1001. ${ }^{a}$ IDs from the $K_{\mathrm{S}}$-selected catalog ${ }^{77} .{ }^{b} M_{*}$, derived from the SED fitting ${ }^{8} .{ }^{c} L_{\mathrm{CO}(1-0)}^{\prime}$, from W18 ${ }^{10} .{ }^{d} \mathrm{CO}$ excitation: $R_{31}=L_{\mathrm{CO}(3-2)}^{\prime} / L_{\mathrm{CO}(1-0)}^{\prime}{ }^{e} M_{\mathrm{dust}}$, derived from the FIR SED fitting with CIGALE $^{45,46}$.

\begin{tabular}{|c|c|c|c|c|c|}
\hline ID & ${ }^{a} \mathrm{ID}_{K_{\mathrm{S}}}$ & $\begin{array}{l}\text { R.A. } \\
\text { J2000 }\end{array}$ & $\begin{array}{l}\text { Decl. } \\
\text { J2000 }\end{array}$ & $z_{\mathrm{CO}(3-2)}$ & $\begin{array}{c}{ }^{b} \log M_{*} \\
M_{\odot}\end{array}$ \\
\hline SB1 & 131077 & 10:00:56.95 & $+02: 20: 17.2$ & 2.494 & $10.93 \pm 0.15$ \\
\hline SB2 & 130891 & 10:00:57.56 & $+02: 20: 11.2$ & 2.512 & $10.83 \pm 0.15$ \\
\hline MS1 & 130949 & 10:00:56.86 & $+02: 20: 08.7$ & 2.503 & $11.36 \pm 0.15$ \\
\hline MS2 & 130901 & 10:00:57.39 & $+02: 20: 10.8$ & 2.507 & $11.35 \pm 0.15$ \\
\hline SFR & ${ }^{{ }^{c}} L_{\mathrm{CO}(1-0)}^{\prime}$ & $L_{\mathrm{CO}(3-2)}^{\prime}$ & ${ }^{d R_{31}}$ & $S_{3.2 \mathrm{~mm}}$ & $\bar{e}{ }^{e} M_{\text {dust }}$ \\
\hline$M_{\odot} \mathrm{yr}^{-1}$ & $10^{10} \mathrm{~K} \mathrm{~km} \mathrm{~s}^{-1} \mathrm{pc}^{2}$ & $10^{10} \mathrm{~K} \mathrm{~km} \mathrm{~s}^{-1} \mathrm{pc}^{2}$ & & $\mu \mathrm{Jy}$ & $10^{8} M_{\odot}$ \\
\hline $1103_{-285}^{+385}$ & $4.9 \pm 0.4$ & $4.10 \pm 0.12$ & $0.84 \pm 0.07$ & $82 \pm 10$ & $16.0 \pm 1.5$ \\
\hline $621_{-161}^{+217}$ & $3.2 \pm 0.3$ & $2.49 \pm 0.09$ & $0.78 \pm 0.08$ & $38 \pm 10$ & $10.8 \pm 2.2$ \\
\hline $528_{-146}^{+201}$ & $2.3 \pm 0.2$ & $1.74 \pm 0.07$ & $0.76 \pm 0.07$ & $62 \pm 12$ & $8.3 \pm 4.8$ \\
\hline $163_{-63}^{+101}$ & $1.8 \pm 0.3$ & $0.79 \pm 0.08$ & $0.44 \pm 0.08$ & $18 \pm 3$ & $4.3 \pm 1.0$ \\
\hline
\end{tabular}


Extended Data Table 2: Gas properties of the four SBs/MSs. The molecular gas masses are estimated based on the CO(1-0) emission line using metallicity-dependent conversion factors, gas-to-dust ratio, and 3.2mm dust continuum emission. ${ }^{a} \mathrm{CO}-$ to- $\mathrm{H}_{2}$ conversion factor $\left(\alpha_{\mathrm{CO}}(Z)\right)^{10}$, calculated based on mass-metallicity relation. ${ }^{b}$ Total molecular gas mass, computed as $M_{\mathrm{gas}, \mathrm{CO}}=\alpha_{\mathrm{CO}}(Z) L_{\mathrm{CO}(1-0)}^{\prime} \cdot{ }^{c} \mathrm{Gas}$ fraction: $f_{\mathrm{gas}}=M_{\mathrm{gas}} /\left(M_{\mathrm{star}}+M_{\mathrm{gas}}\right)$. ${ }^{d}$ Gas depletion time: $t_{\mathrm{dep}}=M_{\mathrm{gas}} / S F R$, which is the inverse of the star formation efficiency (SFE $\left.=1 / t_{\mathrm{dep}}\right) .{ }^{e} M_{\text {gas, GDR }}$, computed based on gas-to-dust ratio. ${ }^{f} M_{\text {gas,3.2mm}}$, computed based on the Rayleigh-Jeans tail dust continuum.

\begin{tabular}{|c|c|c|c|c|}
\hline & SB1 & SB2 & MS1 & MS2 \\
\hline$a_{\alpha_{\mathrm{CO}}(Z)\left(M_{\odot} /\left(\mathrm{K} \mathrm{km} \mathrm{s}^{-1} \mathrm{pc}^{2}\right)\right)}$ & 4.09 & 4.10 & 4.06 & 4.06 \\
\hline${ }^{b} M_{\text {gas, CO }}\left(10^{10} M_{\odot}\right)$ & $20.2 \pm 1.7$ & $13.3 \pm 1.4$ & $9.2 \pm 0.9$ & $7.4 \pm 1.1$ \\
\hline${ }^{c} f_{\text {gas, CO }}$ & $0.70_{-0.06}^{+0.09}$ & $0.66_{-0.07}^{+0.10}$ & $0.29_{-0.06}^{+0.09}$ & $0.25_{-0.06}^{+0.08}$ \\
\hline${ }^{d} t_{\mathrm{dep}, \mathrm{CO}}(\mathrm{Gyr})$ & $0.18_{-0.05}^{+0.07}$ & $0.21_{-0.06}^{+0.08}$ & $0.17_{-0.05}^{+0.07}$ & $0.45_{-0.19}^{+0.29}$ \\
\hline${ }^{e} M_{\mathrm{gas}, \mathrm{GDR}}\left(10^{10} M_{\odot}\right)$ & $17.9 \pm 6.4$ & $12.8 \pm 5.1$ & $7.6 \pm 5.1$ & $4.0 \pm 1.7$ \\
\hline$f_{\text {gas }, 3.2 \mathrm{~mm}}$ & $0.68_{-0.10}^{+0.12}$ & $0.65_{-0.11}^{+0.13}$ & $0.25_{-0.14}^{+0.15}$ & $0.15_{-0.07}^{+0.08}$ \\
\hline$t_{\mathrm{dep}, 3.2 \mathrm{~mm}}(\mathrm{Gyr})$ & $0.16_{-0.07}^{+0.08}$ & $0.21_{-0.10}^{+0.11}$ & $0.14_{-0.11}^{+0.11}$ & $0.24_{-0.14}^{+0.18}$ \\
\hline${ }^{f} M_{\mathrm{gas}, 3.2 \mathrm{~mm}}\left(10^{10} M_{\odot}\right)$ & $22.7 \pm 6.4$ & $10.4 \pm 3.9$ & $17.1 \pm 5.5$ & $5.1 \pm 1.6$ \\
\hline$f_{\text {gas }, 3.2 \mathrm{~mm}}$ & $0.73_{-0.08}^{+0.10}$ & $0.61_{-0.11}^{+0.13}$ & $0.43_{-0.11}^{+0.13}$ & $0.18_{-0.07}^{+0.08}$ \\
\hline$t_{\mathrm{dep}, 3.2 \mathrm{~mm}}(\mathrm{Gyr})$ & $0.21_{-0.08}^{+0.09}$ & $0.17_{-0.08}^{+0.09}$ & $0.32_{-0.14}^{+0.16}$ & $0.31_{-0.16}^{+0.22}$ \\
\hline
\end{tabular}


Extended Data Table 3: Best fit summary of gas kinematic model for the four SBs/MSs. Total ten free parameters and their $1 \sigma$ uncertainties are determined from the GalPAK ${ }^{3 D}$. The parameters are the $x_{\mathrm{c}}, y_{\mathrm{c}}, z_{\mathrm{c}}$ positions, the total flux in the same units as input data cube, the disk half-light radius $r_{1 / 2}$, the turnover radius $r_{\mathrm{v}}$, the inclination Incl., position angle PA, the maximum circular velocity $V_{\text {max }}$, and the intrinsic velocity dispersion $\sigma_{0} .{ }^{a}$ Rotation-to-random motions ratio, calculated from $V_{\max }$ and $\sigma_{0}$. The bottom row is the reduced chi square $\chi^{2}$ in our MCMC fitting.

\begin{tabular}{lcccc}
\hline \multicolumn{2}{c}{ SB1 } & SB2 & MS1 & MS2 \\
\hline Morphological parameters & & & & \\
\hline$x_{\mathrm{c}}$ (pixel) & $16.53 \pm 0.03$ & $17.16 \pm 0.04$ & $15.70 \pm 0.08$ & $16.76 \pm 0.14$ \\
$y_{\mathrm{c}}$ (pixel) & $16.67 \pm 0.02$ & $17.60 \pm 0.03$ & $16.73 \pm 0.06$ & $16.85 \pm 0.10$ \\
$z_{\mathrm{c}}$ (pixel) & $12.75 \pm 0.02$ & $8.10 \pm 0.02$ & $10.22 \pm 0.07$ & $9.85 \pm 0.13$ \\
Flux (Jy beam $\left.{ }^{-1}\right)$ & $2.14 \pm 0.01$ & $1.17 \pm 0.01$ & $1.27 \pm 0.02$ & $0.67 \pm 0.02$ \\
$r_{1 / 2}$ (pixel) & $5.55 \pm 0.04$ & $4.00 \pm 0.05$ & $7.54 \pm 0.13$ & $7.01 \pm 0.32$ \\
$r_{\mathrm{v}}$ (pixel) & $0.54 \pm 0.05$ & $1.89 \pm 0.10$ & $0.06 \pm 0.06$ & $0.60 \pm 0.25$ \\
\hline
\end{tabular}

Orientation of gas disk:

\begin{tabular}{lllll}
\hline Incl. (deg) & $37.37 \pm 0.62$ & $19.30 \pm 1.21$ & $45.72 \pm 1.38$ & $55.58 \pm 2.18$ \\
PA (deg) & $12.32 \pm 0.32$ & $9.94 \pm 0.92$ & $148.68 \pm 0.96$ & $47.75 \pm 2.03$ \\
\hline
\end{tabular}

Kinematic parameters*

\begin{tabular}{lrrrr}
\hline$V_{\max }\left(\mathrm{km} \mathrm{s}^{-1}\right)$ & $419_{-58}^{+21}$ & $497_{-252}^{+123}$ & $327_{-47}^{+89}$ & $245_{-97}^{+211}$ \\
$\sigma_{0}\left(\mathrm{~km} \mathrm{~s}^{-1}\right)$ & $25_{-15}^{+11}$ & $33_{-19}^{+27}$ & $90_{-18}^{+15}$ & $110_{-40}^{+36}$ \\
${ }^{a} V_{\max } / \sigma_{0}$ & $16.8_{-10.4}^{+7.4}$ & $15.0_{-11.3}^{+13.0}$ & $3.6_{-0.9}^{+1.1}$ & $2.2_{-1.2}^{+2.0}$ \\
\hline \multicolumn{7}{c}{} & & & \\
\hline reduced- $\chi^{2}$ & 1.26 & 1.50 & 1.12 & 1.01 \\
\hline
\end{tabular}

* The uncertainties of the kinematic parameters are derived from simulations (see Methods). 

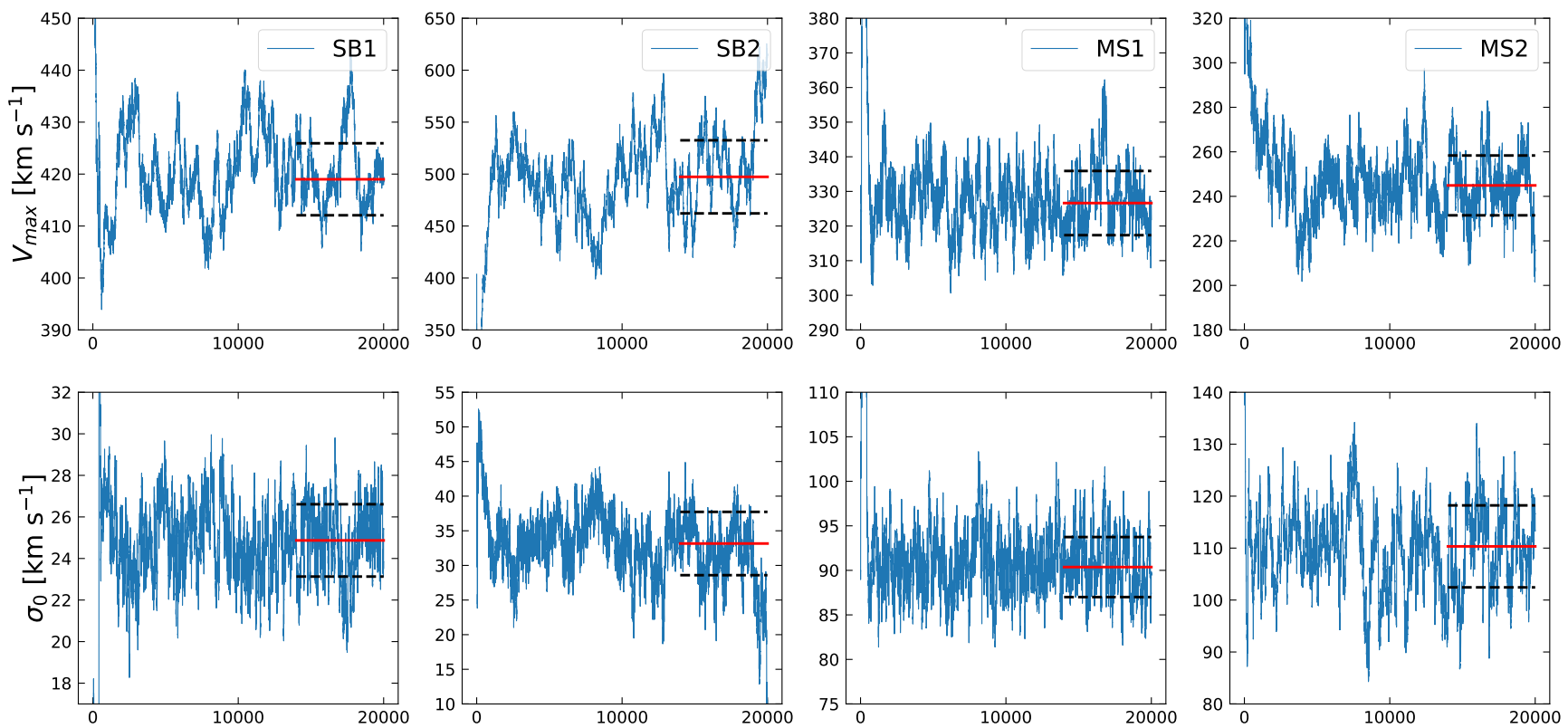

Extended Data Figure 1: Full MCMC chain for 20,000 iterations for the two SBs and two MSs. Each galaxy shows fitting results of rotational velocity and intrinsic velocity dispersion. Red solid lines and black dashed lines refer to the median and the $1 \sigma$ standard deviations of the last $30 \%$ of the MCMC chain.
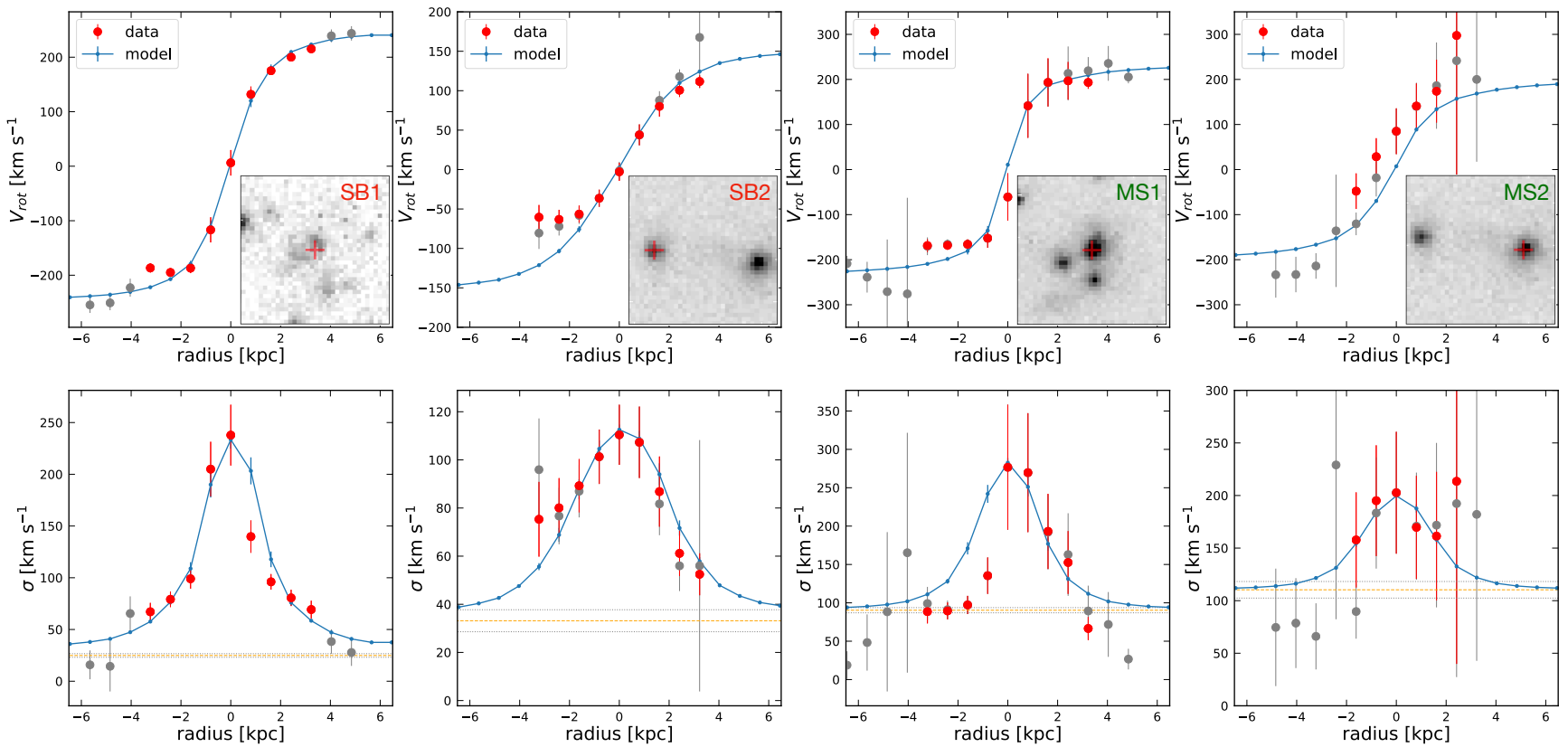

Extended Data Figure 2: Observations (points) and best-fit PSF-convolved models (blue lines) for rotation velocity and total velocity dispersion profiles along the kinematic major axis. Each point was extracted from the spectrum in an aperture whose diameter is equal to the angular resolution $0.31^{\prime \prime}$ and the red one restricted to the spaxels with $\mathrm{S} / \mathrm{N}>3$ in the aperture. Orange and grey lines mark the intrinsic velocity dispersion $\sigma_{0}$ and $1 \sigma$ confidence from the GalPAK ${ }^{3 D}$. The $3.5^{\prime \prime} \times 3.5^{\prime \prime} H S T / F 160 \mathrm{~W}$ images were shown in the bottom right corner with the red crosses denote the corresponding source. Since SB2 and MS2 are close to each other with mixed fluxes, the points at the lowest radius of SB2 and highest radius of MS2 are not fitted well with models. For MS1, the points at the lowest radius are not fitted well with models because of two neighbouring sources at similar redshifts. The $\sigma_{0}$ derived from GalPAK ${ }^{3 D}$ is lower the lowest observed velocity dispersion profile because the latter one is still affected by beam smearing effect even at the large radii probed by our observations. 


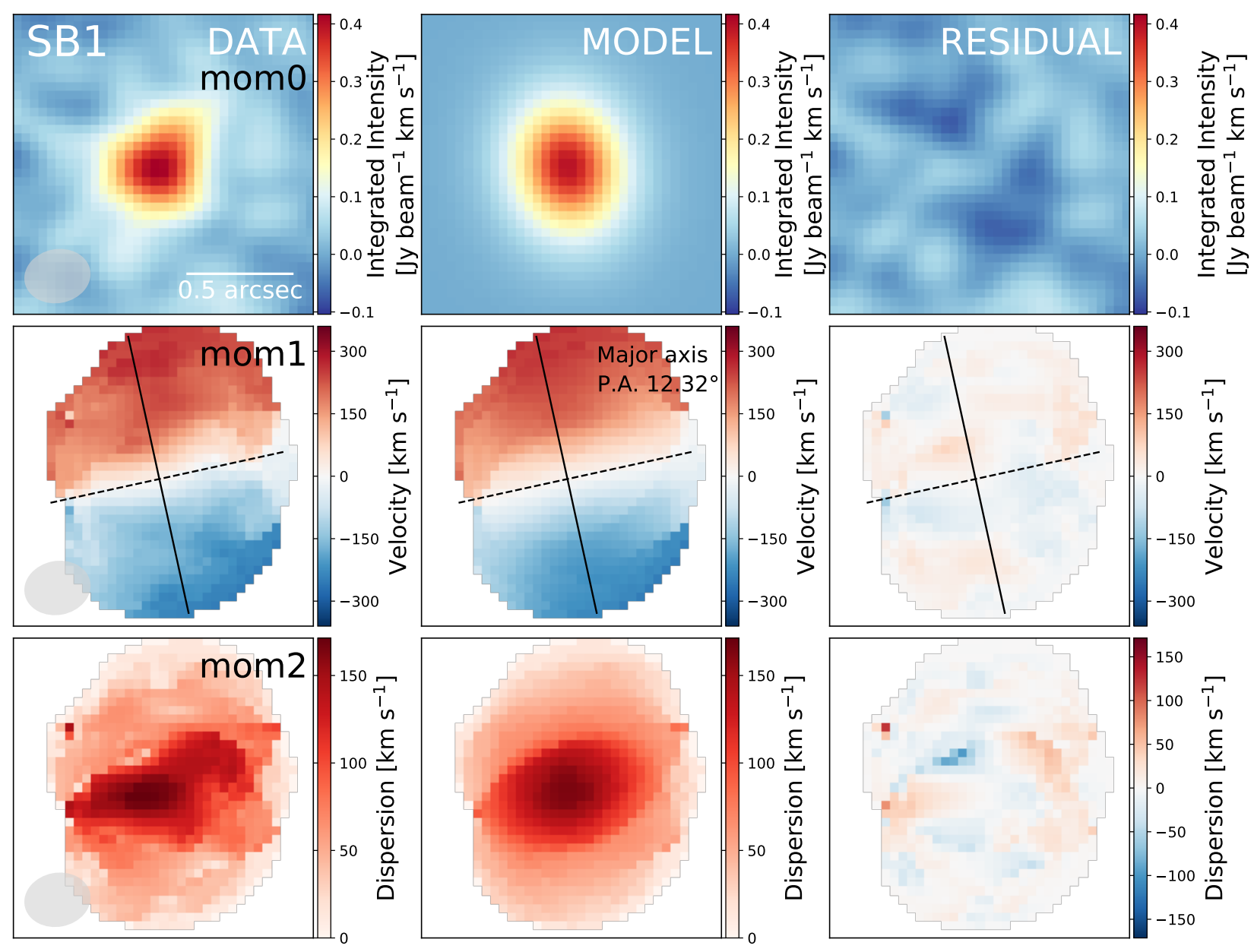

Extended Data Figure 3: Comparison between the data and the best-fit model for the SB1. From top to bottom, it shows velocityintegrated $\mathrm{CO}(3-2)$ flux density (Moment 0 ), velocity field (Moment 1), velocity dispersion (Moment 2) maps for the data (left panel), the best-fit model (middle panel), and the residual after subtracting the model from the data (right panel). Grey filled ellipses in the bottom left corner indicate the angular resolution of $0.31^{\prime \prime} \times 0.25^{\prime \prime}$. Each panel is $1.4^{\prime \prime} \times 1.4^{\prime \prime}$ with the North up and the East to the left. 


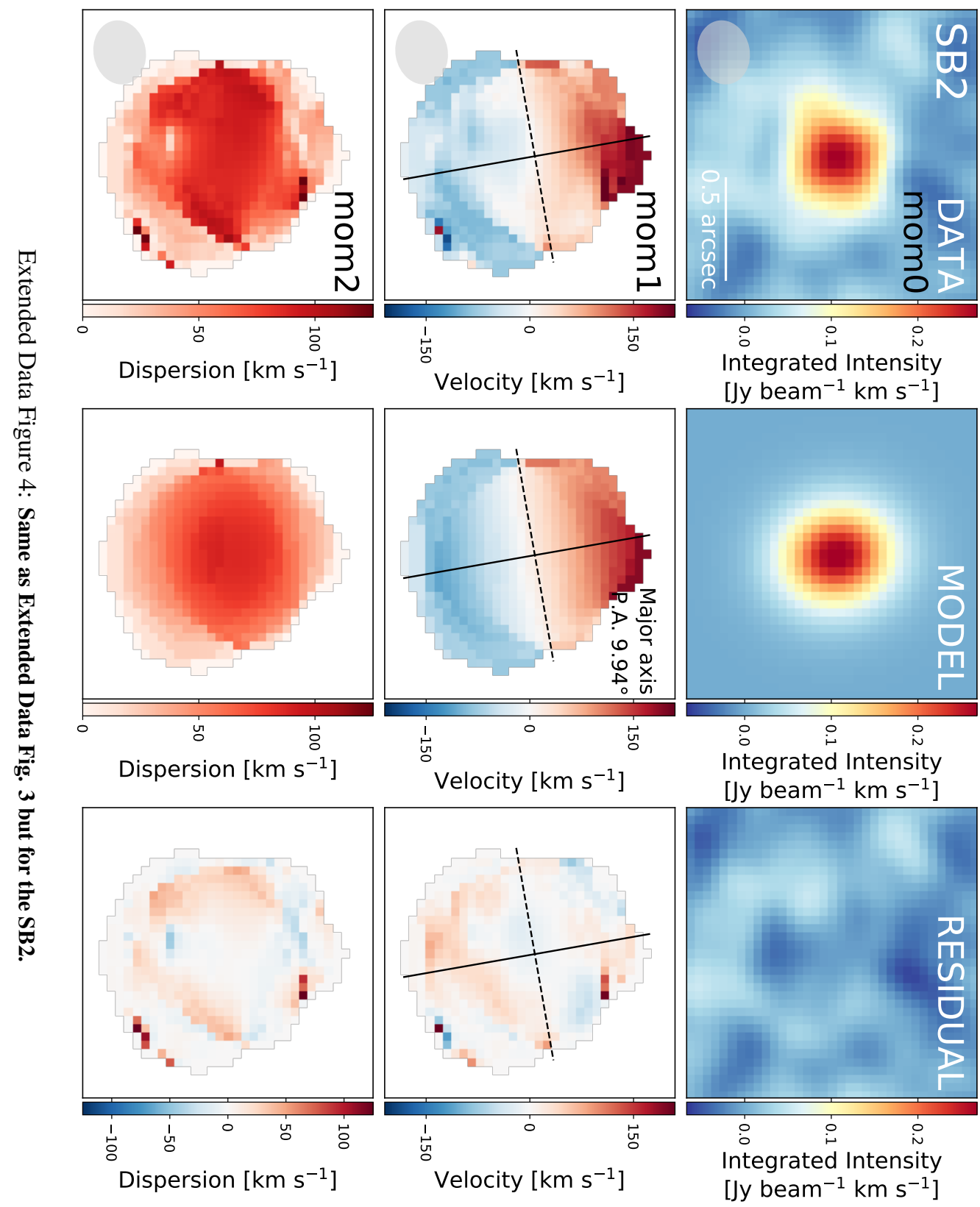




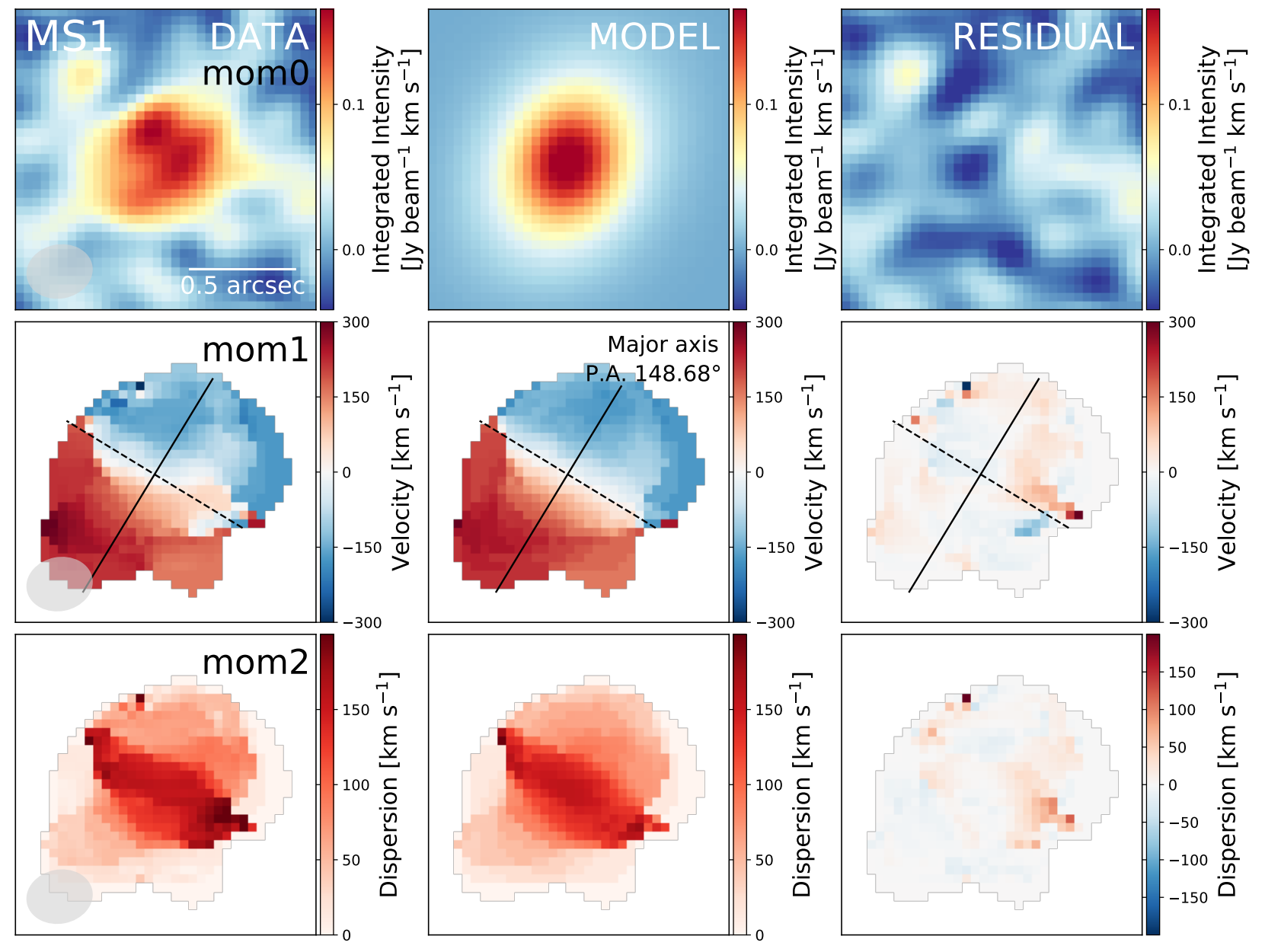

Extended Data Figure 5: Same as Extended Data Fig. 3 but for the MS1. 


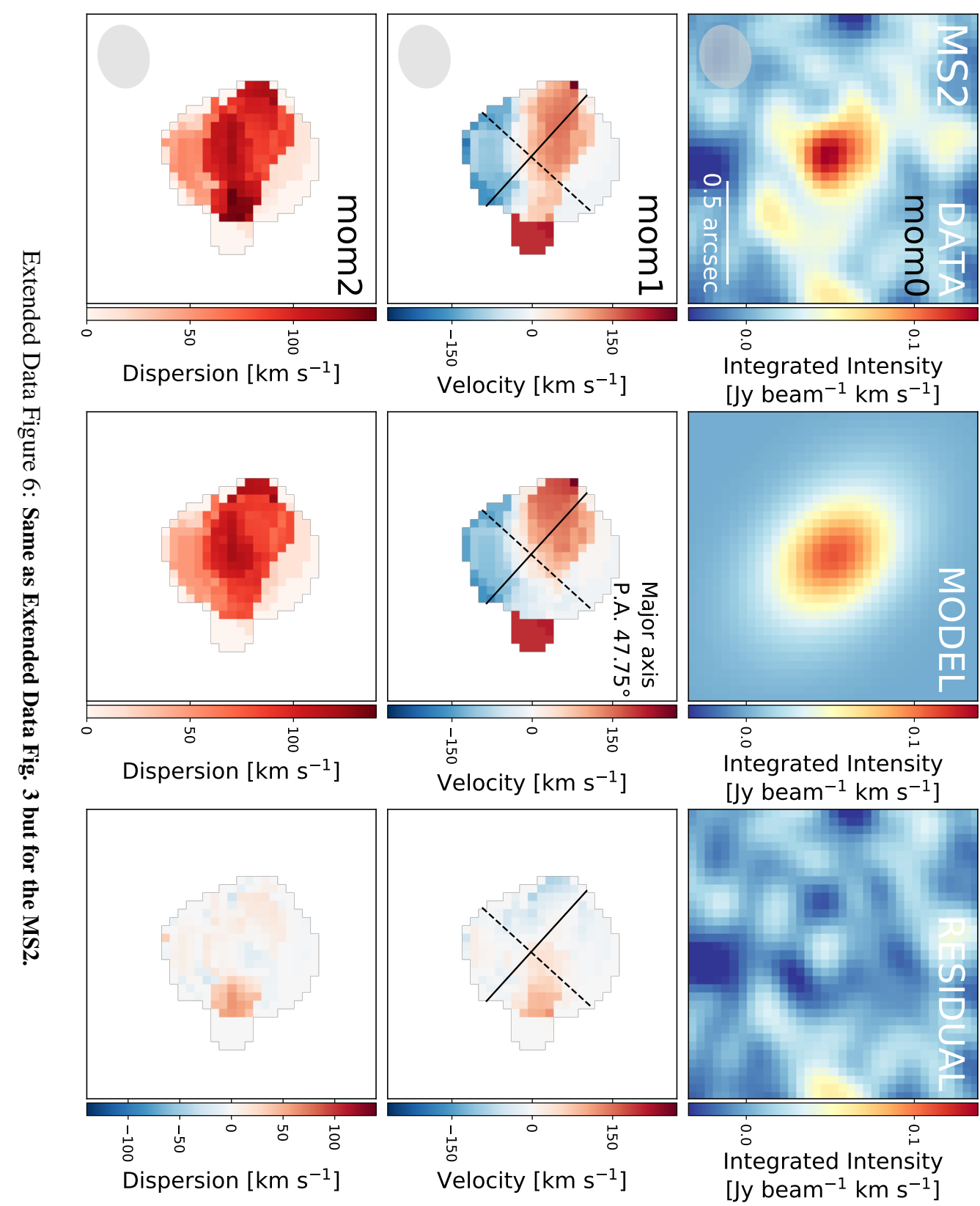




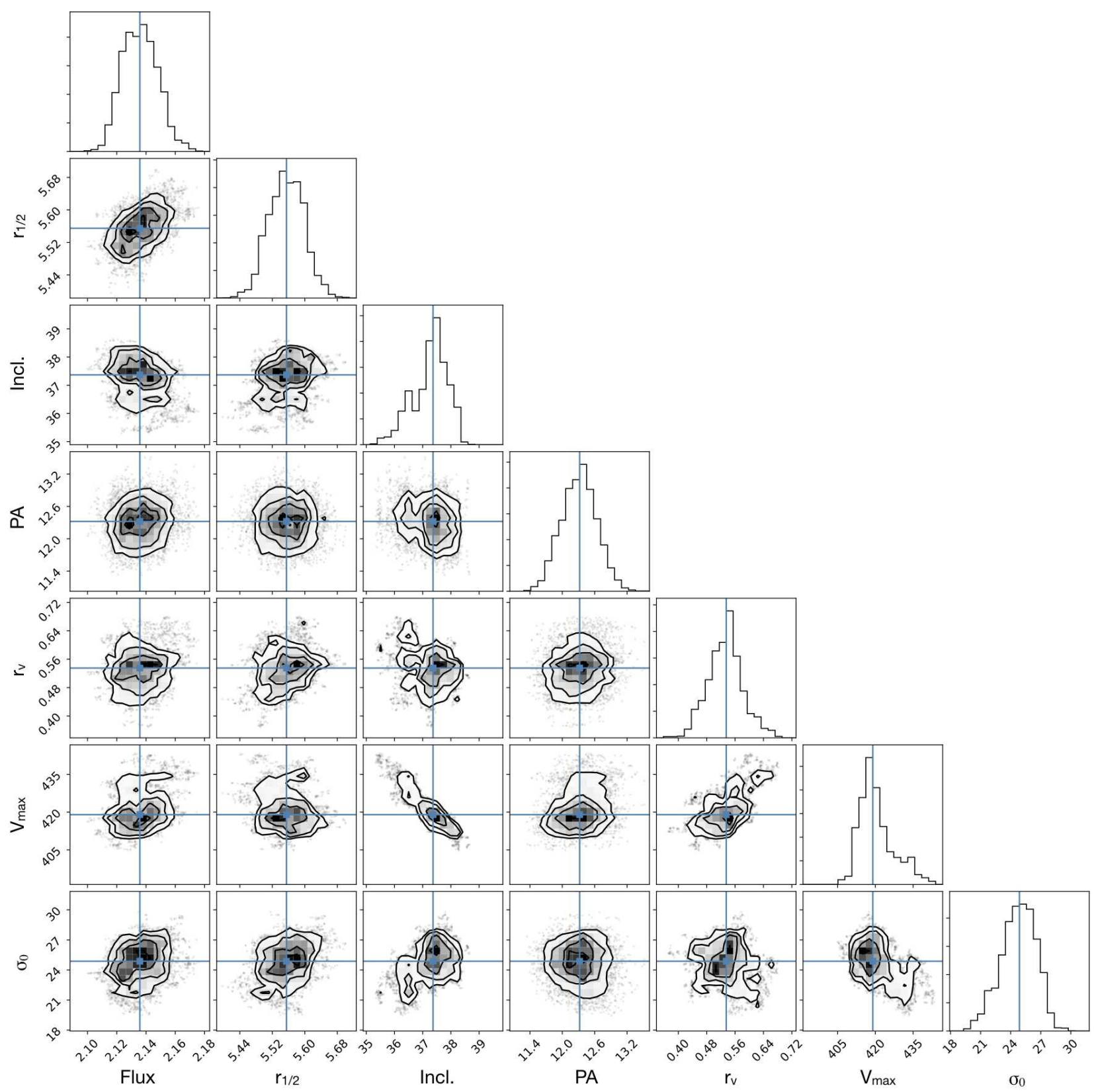

Extended Data Figure 7: MCMC results for the SB1: the panels show the posterior probability distributions of seven model parameters using MCMC sampling with GalPAK ${ }^{3 D}$. Their marginalized probability distribution are shown as histograms. The parameters are the total flux (Flux), the disk half-light radius $\left(r_{1 / 2}\right)$, the inclination angle (Incl.), the position angle (PA), the turnover radius $\left(r_{\mathrm{V}}\right)$, the maximum circular velocity $\left(V_{\max }\right)$, and the intrinsic velocity dispersion $\left(\sigma_{0}\right)$, in the same units as summarized in Extended Data Table 3 The solid blue lines show median values. The black contour corresponds to the $68 \%, 95 \%$, and $99.7 \%$ confidence intervals. 


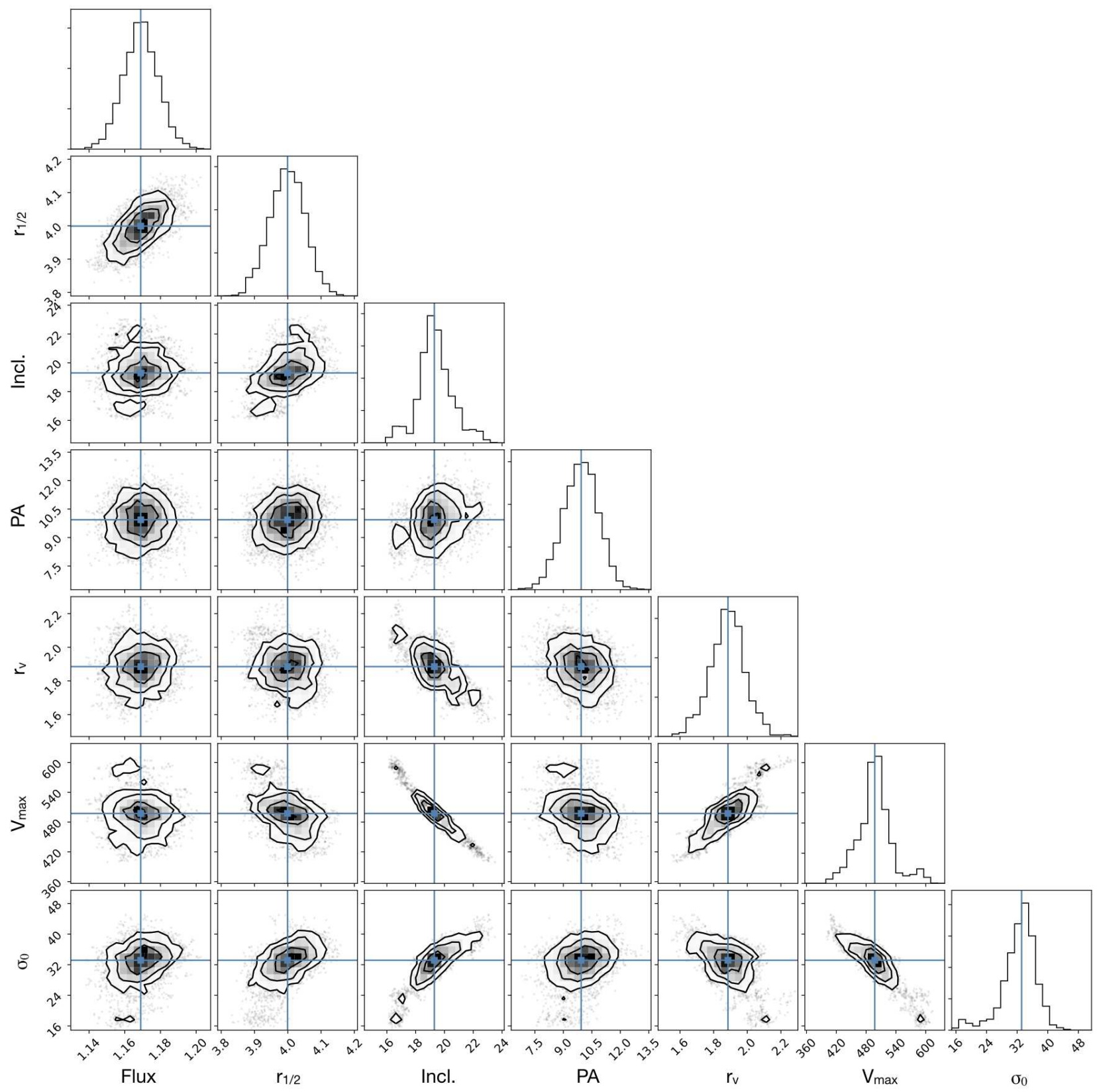

Extended Data Figure 8: Same as Extended Data Fig. 7 but for the SB2. 


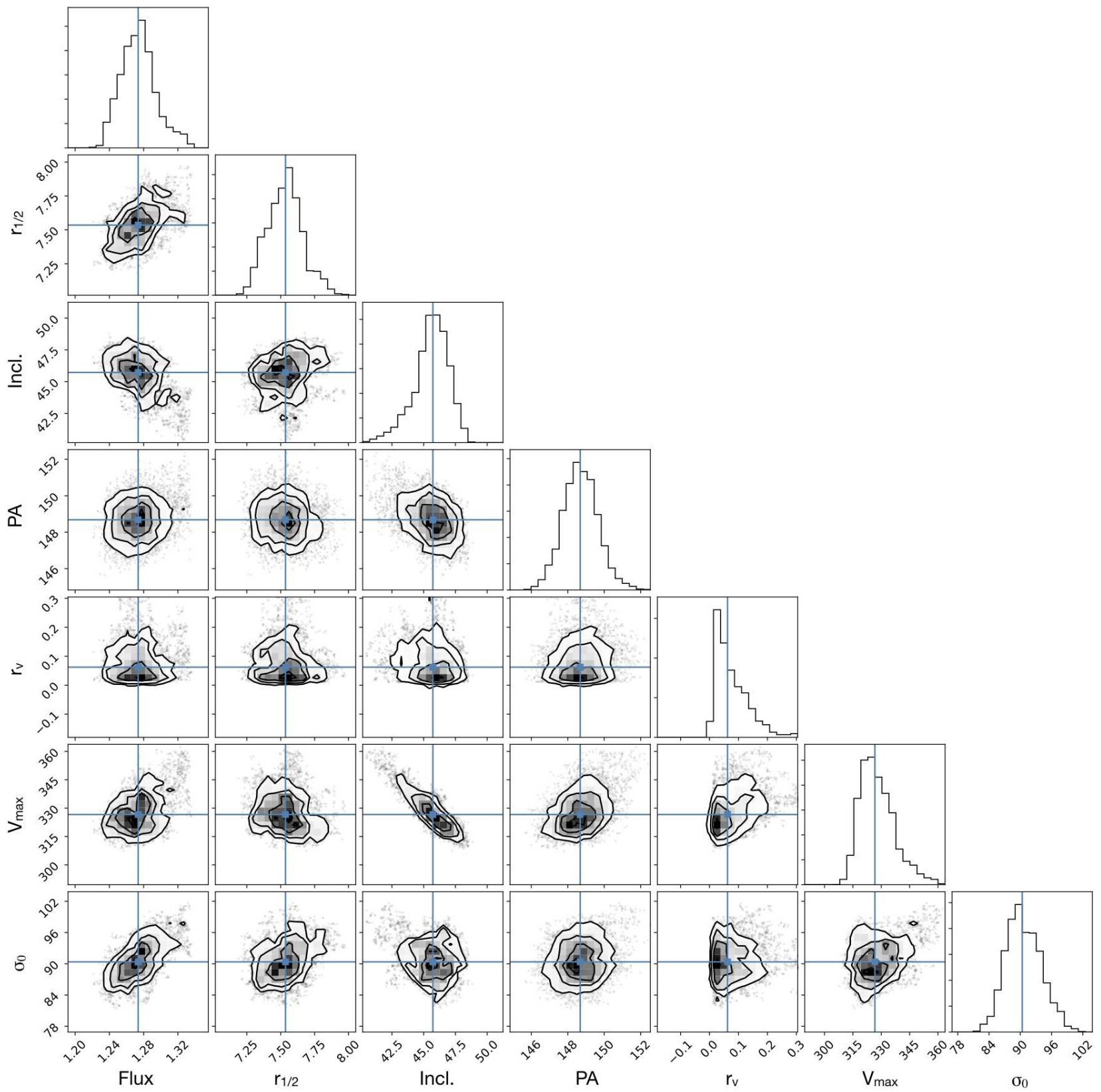

Extended Data Figure 9: Same as Extended Data Fig. 7 but for the MS1. 


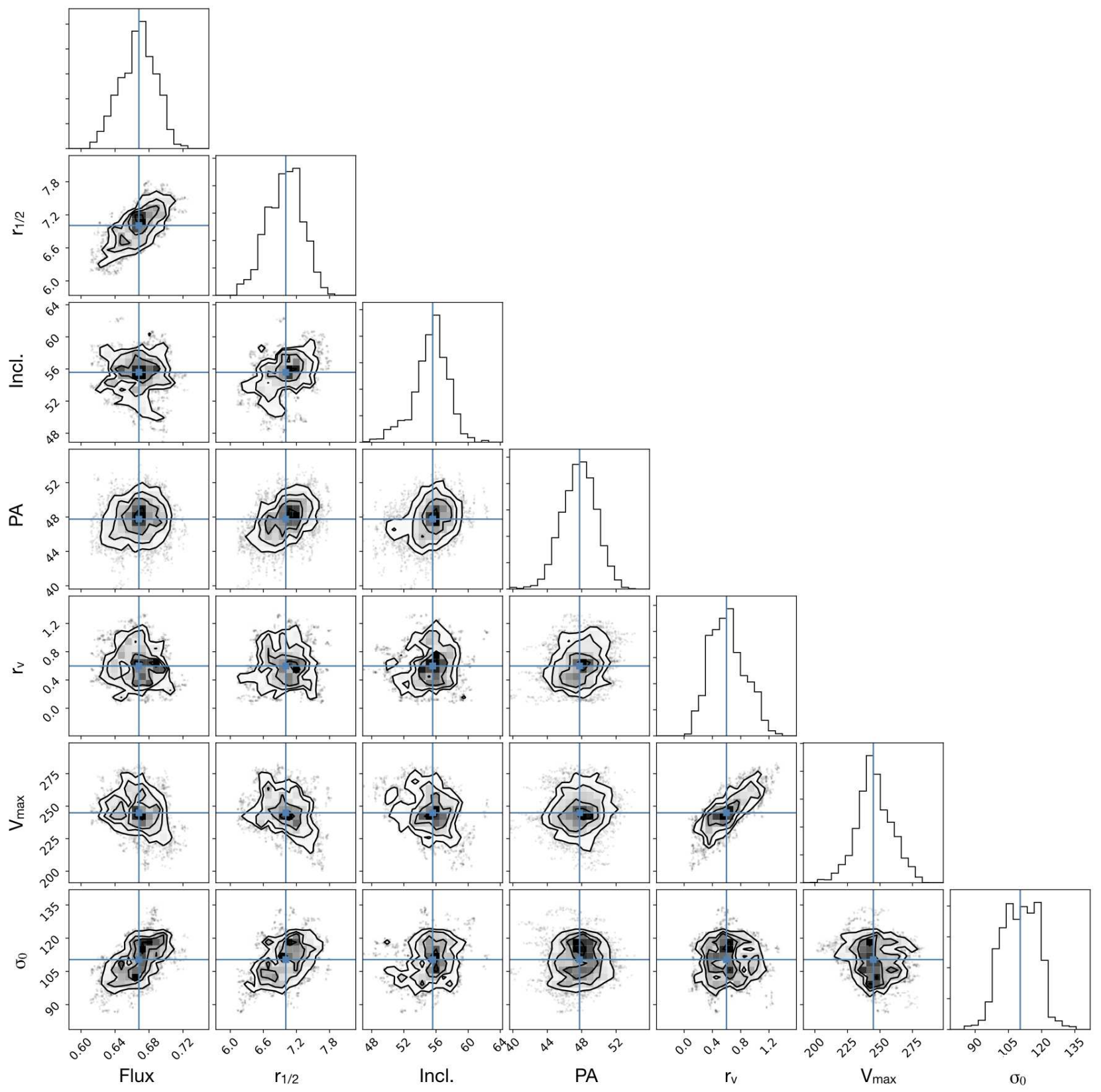

Extended Data Figure 10: Same as Extended Data Fig. 7 but for the MS2. 